\title{
A Pilot Study of an In-Home Multicomponent Exergame Training for Older Adults: Feasibility, Usability and Pre-Post Evaluation
}

\author{
Manuela Adcock ${ }^{1 *}$, Melanie Thalmann ${ }^{1}$, Alexandra Schättin ${ }^{1}$, Federico Gennaro ${ }^{1}$ and \\ Eling D. de Bruin ${ }^{1,2 *}$ \\ ${ }^{1}$ Institute of Human Movement Science and Sport, Department of Health Sciences and Technology, ETH Zürich, Zurich, \\ Switzerland, ${ }^{2}$ Division of Physiotherapy, Department of Neurobiology, Care Sciences and Society, Karolinska Institute, \\ Stockholm, Sweden
}

Aging is associated with sensory, motor and cognitive impairments that may lead to reduced daily life functioning including gait disturbances, falls, injuries and mobility restrictions. A strong need exists for implementing effective evidence-based interventions for healthy aging. Therefore, the aim of this study was to (i) evaluate the feasibility and usability of an in-home multicomponent exergame training and (ii) explore its effects on physical functions, cognition and cortical activity. Twenty-one healthy and independently living older adults were included (11 female, $74.4 \pm 7.0$ years, range:

OPEN ACCESS

Edited by:

Philip P. Foster,

University of Texas Health Science Center at Houston, United States

Reviewed by:

Katie Palmer, Catholic University of the Sacred Heart Rome, Italy Dafna Merom Western Sydney University, Australia

${ }^{*}$ Correspondence: Manuela Adcock manuela.omlin@hest.ethz.ch Eling D. de Bruin eling.debruin@hest.ethz.ch

Received: 19 March 2019 Accepted: 24 October 2019 Published: 22 November 2019

Citation: Adcock M, Thalmann M,

Schättin A, Gennaro F and de Bruin ED (2019) A Pilot Study of an In-Home Multicomponent Exergame Training for Older Adults: Feasibility, Usability and Pre-Post Evaluation. Front. Aging Neurosci. 11:304 doi: 10.3389/fnagi.2019.00304 65-92 years) and performed 24 trainings sessions (each 40 min) over eight weeks. The first part was conducted in a living lab (home-like laboratory environment), the second part at participants' home. The multicomponent exergame included Tai Chiinspired exercises, dance movements and step-based cognitive games to train strength, balance and cognition. Attendance and attrition rates were calculated and safety during training was evaluated to determine feasibility. Participants rated the usability of the exergame (System Usability Scale) and reported on their game experience (Game Experience Questionnaire). Physical and cognitive functions and cortical activity (resting state electroencephalopathy) were assessed pre and post intervention. Results showed a high training attendance rate for the living lab and the home-based setting (91.7 and $91.0 \%$, respectively) with a rather high attrition rate (28.6\%, six drop-outs). Half of the drop-out reasons were related to personal or health issues. System usability was rated acceptable with a mean score of 70.6/100. Affective game experience was rated favorable. Significant improvements were found for minimal toe clearance, shortterm attentional span, and information processing speed $(0<0.05)$. No significant pre-post differences were found for cortical activity. To summarize, the exergame is generally feasible and usable for healthy older adults applied in an in-home setting and provides an overall positive emotional game experience. Nevertheless, flawless technical functionality should be a mandatory consideration. Additionally, the training might have potential positive influence on specific functions in older adults. However, the efficacy has to be evaluated in a future randomized controlled trial assessing the behavioral and neuroplastic changes in a larger population after a longer training period.

Keywords: exergame, healthy aging, fall prevention, elderly, feasibility, usability 


\section{INTRODUCTION}

As life expectancy is increasing, the number of people aged 60 years and older is rapidly growing. According to the World Health Organization, the number of older adults aged 60 years and above was about $9 \%$ of the worldwide population in 2015 and will more than double to $22 \%$ by 2050 (National Institutes of Health, 2011). Considering the rapid growth in the elderly population, the maintenance and improvement of health and independence of older adults is important for social and economic reasons. A major focus should aim to prevent problems that cause health restrictions and morbidity, e.g., fall events (Masud and Morris, 2001; Rubenstein, 2006). Falling can lead to serious adverse consequences as injuries, movement restrictions, and part to full loss of independence (Peel, 2011). In addition to the physical consequences, falls can also lead to fear of falling resulting in further activity restriction, social isolation, feelings of helplessness, depression, and a general decrease in well-being (Jung, 2008; Peel, 2011). Considering the significant impact on individual lives and on healthcare costs, a strong need exists to develop and examine interventions that aim to support healthy aging and prevent falls in older adults.

In order to successfully support healthy aging and prevent falls, risk factors of impaired daily life functioning including falling have to be identified. Age- and lifestyle-associated degenerative changes in motor and sensory systems are linked to impaired daily life activities e.g., gait performance (Willett et al., 2006). The often described decline in muscle strength and loss of balance are discussed as potential risk factors for gait disturbances and falling (Rubenstein, 2006; Taylor et al., 2012). Exercise interventions aiming to improve physical functions, such as strength or balance, have been shown to reduce fall rates and risks in older adults (Sherrington et al., 2008; ElKhoury et al., 2013; Karlsson et al., 2013; Schoene et al., 2014). However, walking, as most daily life activities, requires physical and cognitive resources (Holtzer et al., 2006; de Bruin and Schmidt, 2010; Mirelman et al., 2012). A continuous interaction of physical and cognitive functions is mandatory for safe gait and intact daily life functioning in general (Hausdorff et al., 2005; Delbaere et al., 2010). Therefore, more recently increased attention is paid to cognition as a further aspect of fall prevention programs. The aging brain undergoes several structural and functional changes which can lead to a decline in cognitive functions (Rossini et al., 2007; Gunning-Dixon et al., 2009). The reduced cognitive resources might contribute to impaired daily life functioning and fall events due to an impaired physicalcognitive interplay. Thus, a combined physical-cognitive training is important for effective and holistic support of healthy aging and fall prevention (Pichierri et al., 2011; Bamidis et al., 2014; Eggenberger et al., 2015). Promising options for simultaneous training of physical and cognitive functions are video game-based physical exercises, or so-called exergames (de Bruin et al., 2010).

The term exergaming is a portmanteau composed of "exercise" and "gaming" and is defined as "any type of video game play requiring players' whole body movements" (Gerling and Mandryk, 2014). More recently, exergames have gained increasing popularity in supporting healthy aging and fall prevention (Van Diest et al., 2013, 2016). This might be related to the technological development but also to the multifaceted potential of exergames. Poor adherence is described for numerous existing conventional exercise interventions (Choi et al., 2017). Such barriers, that hinder the attendance at physical activities and exercise interventions, can be related to personal (behavioral) factors such as motivation, personal beliefs, lack of time or feeling uncomfortable in social settings (e.g., in training classes) (Schutzer and Graves, 2004; Yardley et al., 2006; Franco et al., 2015). Furthermore, environmental factors including distance to an exercise facility, poor access to transportation options and participation costs may lead to low(er) exercise compliance as well (Schutzer and Graves, 2004; Yardley et al., 2006; Franco et al., 2015). Many of these barriers may be counteracted by exergames as they provide enjoyable and, therefore, motivating gameplay (Hoffmann et al., 2015; Pirovano et al., 2016). Furthermore, exergames can be applied in diverse settings. For example, the application in an in-home setting facilitates access without obstructions. Moreover, exergames can be adapted to specific purposes and target populations, which might be important when using interventions for public health and disease prevention. To summarize, advantages of exergames compared to conventional exercises are: (1) Exergames can imply simultaneous training of physical and cognitive functions (thereby being closer to daily life requirements) (Van Diest et al., 2013); (2) Users get motivated through an engaging and interactive training (Choi et al., 2017); (3) Training principles (e.g., feedback, progression, task variability) can be implemented in the exergame structure (Healy et al., 2014; Skjæret et al., 2016); (4) Exergame training can be conducted in diverse settings, e.g., at home. Recent studies have shown high acceptability of homebased exergame training for older adults after a mild injury and for elderly living in a nursing home (Valiani et al., 2017; Lauzé et al., 2018). Thus, developing an exergame for an in-home training solution might be a promising strategy to overcome some of the barriers for training attendance in older adults.

Considering the theoretical background from human movement science and neuropsychology together with the art of game design, we developed the Active@Home exergame to support healthy aging including fall prevention. Following a user-centered design approach, the needs and requirements of older adults as target users have been incorporated (Brox et al., 2017). The Active@Home exergame includes strength, balance, and cognitive training components and aims at motivating older adults toward a more active lifestyle. A first pilot-study explored the usability of the newly developed exergame prototype in a laboratory setting where training sessions were closely supervised. The results showed high usability of the exergame prototype and, furthermore, delivered some suggestions for improvements and adaptations in a next iterative development step. The information gathered in the first exploratory trial were, therefore, used to develop the exergame intervention for application at older adults' homes by following a framework for the design and evaluation of complex interventions (Campbell et al., 2000). Therefore, the primary aim of this study was to evaluate the feasibility and usability of the modified Active@Home 
exergame in an in-home setting and to gain information from older adults about the training system. A secondary aim was to explore whether this intervention effects on physical and/or cognitive functions as well as on cortical activity at rest.

\section{MATERIALS AND METHODS}

\section{Study Design and Participants}

This study is an exploratory trial, a phase II study according to Campbell and colleagues, using a single arm pre-post testing design (Campbell et al., 2000). Recruitment of participants was conducted in March 2018 through public advertisements in a local newspaper (Höngger Zeitung, Zurich, Switzerland) and through contacting the pensioner community ETH Zurich (PVETH, Zurich, Switzerland). Assessments and the first half of the intervention were performed at ETH Hönggerberg (Zurich, Switzerland). The second half of the intervention took place at participants' home. The intervention period started in April 2018 and lasted until the beginning of June 2018. Measurements were conducted before and after the intervention. Ethical approval (protocol number EK 2018N-07) was granted by the ETH Zurich Ethics Committee (Zurich, Switzerland). All participants were fully informed prior to participation and signed an informed consent form according to the Declaration of Helsinki before conducting any measurement.

For this trial, healthy and independent living older adults aged 65 years and above were recruited. To evaluate the eligibility of the potential participants, they were screened using the Mini Mental State Examination (MMSE) to assess cognitive status. In addition, the participants completed a health questionnaire including anthropometric data and questions about their health, medical history and physical activity level. Participants fulfilling all of the following inclusion criteria were eligible for the study: (1) age $\geq 65$ years, (2) living independently, (3) healthy (self-reported), (4) able to stand at least for $10 \mathrm{~min}$ without assistance, (5) access to a TV with HDMI connection. Participants exhibiting at least one of the following criteria were excluded from the study: (1) mobility impairments that prevent from training participation, (2) severe and uncontrolled health problems (e.g., recent cardiac infarction, uncontrolled diabetes or hypertension), (3) orthopedic disease that prevents from training participation, (4) neurological disease (e.g., history of stroke or epilepsy, Parkinson's disease), (5) Alzheimer disease or other forms of dementia, (6) acute severe, rapidly progressive or terminal illness, (7) cognitive impairments (MMSE $\leq 23$ points), (8) intake of any psychoactive substances (e.g., neuroleptics, antidepressants), (9) high alcohol, caffeine or nicotine consumption. The minimal intended study sample size of 20 participants starting with the training program was based on similar feasibility studies (Wuest et al., 2014; Nawaz et al., 2016), the "rule of 12" for continuous variables (Moore et al., 2011) and the expected compliance with the intervention (Nyman and Victor, 2012). The "rule of 12 " recommends to include at least 12 participants for pilot studies for providing valuable preliminary information (Moore et al., 2011).

\section{Exergame Intervention}

The hardware of the Active@Home exergame consists of four inertial measurement units (IMUs) providing both accelerometer and gyroscope assessments. For movement evaluation, participants wore the IMUs at wrists and ankles attached with a silicone slap band. The IMUs were connected via Bluetooth to a HDMI dongle that was inserted into a television (TV) and ran the exergame software. The game interface was presented on the TV screen. The story of the exergame was about traveling in Europe and to train in several different cities (London, Paris, Amsterdam, Rome, Porto, and Zurich). Exercises were instructed by a cartoon-based instructor and were accentuated with background music (Tabei et al., 2017). The training content of the Active@Home exergame was based on current recommendations for exercises to prevent falls in older adults (American Geriatrics Society, British Geriatrics Society, and American Academy of Orthopaedic Surgeons Panel on Falls Prevention, 2001; Nelson et al., 2007; Chodzko-Zajko et al., 2009; Panel on Prevention of Falls in Older Persons et al., 2011; Sherrington et al., 2011; Bamidis et al., 2014). The exergame included Tai Chi-inspired exercises, dance movements as well as step-based cognitive games aiming to train strength, balance, and cognition, respectively. Tai Chi-based training has been discussed as a suitable exercise for fall prevention ( $\mathrm{Hu}$ et al., 2016; Zhao and Wang, 2016). Tai Chi is a functional training involving bilateral and multidirectional movements (Xu et al., 2008), requiring whole body coordination (Hackney and Wolf, 2014), and improving core and lower limbs muscle strength; e.g., m. iliopsoas, m. quadriceps femoris and m. tibialis anterior (Solloway et al., 2016). In the Active@Home exergame, four to five Tai Chi levels were implemented in each city with increasing difficulty. In each Tai Chi level, the participant performed three series of about 10 exercise repetitions, with a rest of $20 \mathrm{~s}$ between series. This resulted in a duration of 2-3 min for each Tai Chi level. During the $20 \mathrm{~s}$ rest between series, participants were asked questions about the city in which they were exercising to entertain them. The initial positions of the Tai Chi-inspired exercises included squats, plies, lunges and single-leg stances. Furthermore, the Active@Home exergame contained dance exercises based on several different dancing styles (Bachata, Salsa, Cha-Cha-Cha, Waltz, Jive, and Disco Fox). Conducting rapid and well-directed steps has been shown to be an effective training for fall prevention (Kattenstroth et al., 2013; Okubo et al., 2016). In each city of the exergame, one dancing style was trained in three levels of difficulty. Each dance level lasted around $3 \mathrm{~min}$. Additionally, step-based cognitive games were included in the Active@Home exergame focusing on specific cognitive functions such as selective attention, divided attention, mental flexibility, inhibition/interference control, and working memory. Deficits in these cognitive functions contribute to gait disturbances and falls (Amboni et al., 2013). By stepping forward, backward, and to the right or left side, the cognitive games were played and controlled (Figure 1). Each city of the exergame included three different step-based cognitive games, 


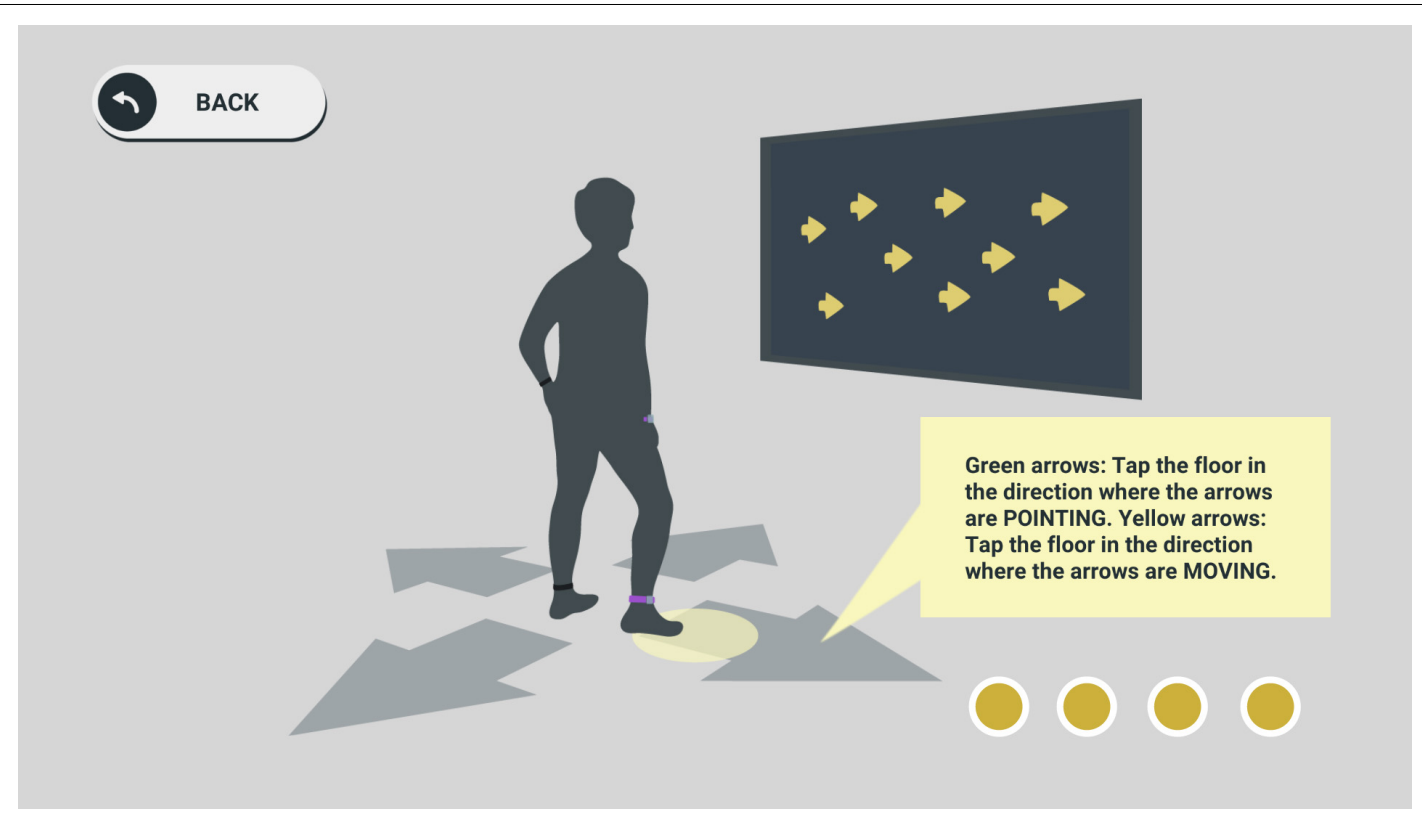

FIGURE 1 | Step-based cognitive training. The Active@Home exergame included step-based exercises to train specific cognitive functions (a task for inhibition training is shown in the picture). By stepping forward, backward, and to the right or left side, these games were played and controlled. The IMUs worn at ankles evaluated the stepping performance.

each game lasting between 1 and $2 \mathrm{~min}$. Moreover, the exergame implemented some basic training principles (feedback, optimal load, progression, variability) which are important for effective training and for reaching the training goal of improvements in physical and cognitive functioning (Healy et al., 2014). A feedback system was included with a real-time color code for achieved performance (red color = "bad performance"; orange color $=$ "moderate performance"; green color $=$ "good performance") and performance scores during and after each exercise. In the cognitive games, the visual feedback system described above was augmented with an auditory feedback system providing sounds to indicate the correctness of the answer. To ensure optimal load and progression, several difficulty levels for Tai Chi-inspired and dance exercises were developed in each city. Progression was reached through more complex movements in the Tai Chi-inspired exercises (e.g., additional arm movements, upper body rotations, increased range of motion, longer time in unstable position) and through additional weights (e.g., filled water bottles), while faster and more complex motion sequences were performed in dance exercises. To increase difficulty in step-based cognitive training, more complex games were used requiring e.g., faster reaction times or memorization of additional information. Moreover, the training exercises got more challenging from city to city with a predefined city order of progressive difficulty. Training variability was guaranteed through a high diversity of exercises in the different cities of the exergame.

From April to June 2018, each participant performed 24 training sessions in 8-10 weeks (a maximum of two weeks holiday interruption was allowed). Each week, three training sessions were performed, which were scheduled individually from Monday to Friday with a guideline of no more than one training session per day. Each session consisted of 40 min training with the Active@Home exergame including 15 min of Tai Chiinspired training, $15 \mathrm{~min}$ of dance exercises, and $10 \mathrm{~min}$ of step-based cognitive games. It was recommended to play through all levels of one city before switching to the next city whereas the order of the cities was predefined based on progressive difficulty. Additionally, before changing the level, one level should have been trained at least in two sessions. The current difficulty level should always provide an optimal challenge avoiding underor overload. The training intensity was thereby individually adapted to target a moderate training intensity level (ChodzkoZajko, 2014). Intervention characteristics as frequency, duration, and training intensity were based on recommendations for fall prevention in elderly (Nelson et al., 2007; Paterson et al., 2007; Chodzko-Zajko et al., 2009) and on studies showing positive training effects of exergame training in older adults (Law et al., 2014).

Participants trained 3-4 weeks in the home-like living lab at ETH Hönggerberg (Zurich, Switzerland) and 4-5 weeks at their home. The training sessions in the living lab were supervised by two postgraduate students supporting the participants if needed and systematically observing them while using and training with the exergame. During the intervention period at home, participants were called weekly to provide help when needed.

\section{Primary Outcomes}

A mixed method approach in form of a combination of quantitative and qualitative data collection was used to evaluate the primary outcome of feasibility and usability of the Active@Home exergame (Nawaz et al., 2016). Questionnaires 
were completed by participants after their last home training session of the intervention period (post-measurement).

\section{Feasibility}

Feasibility was assessed through compliance with the training intervention as well as by evaluation of the safety during training. An attendance protocol was used to record the number of performed training sessions. During the intervention period in the living lab, the protocol was filled in by the supervisors after each training session, whereas participants were instructed to protocol the training sessions during the intervention period at home. Additionally, during weekly calls by one of the supervisors, the protocoling procedure was checked. The adherence rate was calculated using the number of performed training sessions as percentage of the maximum possible training sessions (De Bruin et al., 2011; Wuest et al., 2014). A 70\% attendance rate (17 visited out of 24 total training sessions planned) was considered "being adherent" to the training program (De Bruin et al., 2011; Nyman and Victor, 2012). The adherence rate was calculated for the two intervention periods (living lab, home) separately and in total. For attrition, the number of participants lost during the trial was recorded (drop-outs) and calculated as a percentage of the total sample size. Considering the median rate for attrition in fall prevention interventions for clinical trials, a $10 \%$ attrition rate (two drop-outs) was regarded acceptable (Nyman and Victor, 2012). Drop-outs were not considered in the calculation of the adherence rate. Moreover, reasons for non-adherence and dropouts were recorded.

After the intervention, participants answered five questions related to safety including dizziness or pain during training, "critical moments" e.g., tripping, slipping or swaying, fear of falling or fall events. Safety questions were answered with "yes" or no" (in case of dizziness/pain also rating of level).

\section{Usability}

The System Usability Scale (SUS) is a validated and reliable scale for evaluating subjective usability of newly developed devices and systems and includes 10 items rated on a 5-point Likert scale ( 0 = "strongly disagree" to 4 = "strongly agree") (Brooke, 1996; Vaziri et al., 2016). The sum of all item scores was multiplied with 2.5 and led to the SUS score theoretically ranging between 0 and 100. Higher scores indicate better usability (Brooke, 1996). Based on the verbal categorization rate of Bangor (Bangor et al., 2009), a SUS score $\geq 70$ was expected for an "acceptable system". An additional question was added at the end of the SUS, asking participants about their general opinion of the Active@Home exergame. This question was also rated on a 5-point Likert scale ( 0 = "I don't like it" to $4=$ "I like it a lot") and the mean was calculated over all participants.

The Game Experience Questionnaire (GEQ) assesses seven categories of subjective game experience (competence, immersion, flow, tension, challenge, negative affect, and positive affect) (Hoffmann et al., 2015; Chesham et al., 2017). Competence implies feelings of being successful, strong or skillful in the game. Immersion includes the interest and pleasure of a player in the game. Flow summarizes the feelings of being deeply concentrated and absorbed, forgetting time, and losing connection to the world outside the game. Tension includes feelings of annoyance, frustration, and pressure. Challenge implies feelings of being stimulated and challenged. Negative affect summarizes feelings related to a bad mood and boredom, whereas positive affect includes feelings of happiness and enjoyment. The GEQ has been applied in several studies evaluating game experience of exergames for elderly (Liukkonen et al., 2015; Merriman et al., 2018). The GEQ core module, which was used in this study, includes in total 42 items rated on a 5-point Likert scale ( $0=$ "not at all" to $4=$ "extremely"). It was analyzed by calculating the average rating for each of the aforementioned categories (Ijsselsteijn et al., 2008). Two categories involved only negative coded items (tension and negative affect) leading to reverse evaluation (Chesham et al., 2017).

Observations by supervisors and feedback by participants were recorded on a usability protocol. The usability protocol was structured in six categories: (1) functionality and interaction with the system, (2) IMUs, (3) design, (4) training principles, (5) exercises, and (6) emotions, each category separated in positive and negative aspects. The participants were instructed (but not forced) to "think aloud" and mention all thoughts that came to their mind during exergame training (Lewis and Rieman, 1993). Their comments and the observations by the supervisors (during the intervention period in the living lab) were recorded in writing on the usability protocol. Furthermore, the protocol included feedback from participants collected during weekly calls (during the intervention period at home).

\section{Secondary Outcomes}

As secondary outcomes, physical and cognitive functions as well as electroencephalographic cortical activity were measured before and after the intervention period (pre-measurement and post-measurement).

\section{Physical Functions}

Parameters of gait kinematics (speed, cadence, stride length, and minimal toe clearance) were assessed using the Physilog ${ }^{\circledR} 5$ IMU (Gait Up Sàrl, Lausanne, Switzerland), which has been shown to reliably and validly measure gait performance (Aminian et al., 1999; Dubost et al., 2006; de Bruin et al., 2007). The Physilog ${ }^{\circledR} 5$ IMUs were attached to the top of the right and left forefoot of participants using elastic straps. For further analysis, data was transferred to the computer via USB port. A walking protocol involving at least 50 gait cycles was applied (König et al., 2014). Participants walked a straight distance of $80 \mathrm{~m}$ under two conditions: (1) single-task condition (ST): participants were instructed to walk at preferred speed without talking; (2) dual-task condition (DT): participants had to walk at preferred speed and simultaneously count backwards (cognitive task) in steps of seven from a randomly given number between 200 and 250. In this condition, participants were asked to count out loud and perform both tasks concurrently and not to prioritize one task above the other. This is a common method to measure multitasking capabilities (Eggenberger et al., 2015; Schättin et al., 2016). Two walking steps for initiation and termination were discarded in order to analyze steady state walking (Jian et al., 1993). Speed [m/s], cadence [steps/min], stride length [m], and 
minimal toe clearance $[\mathrm{cm}]$ were evaluated and expressed as mean values of both legs in the two walking conditions. For each parameter, the dual-task cost (DTC) of walking was calculated as a percentage of loss of the DT relative to the ST condition according to the formula: DTC $[\%]=(\mathrm{ST}-\mathrm{DT}) / \mathrm{ST} \times 100$ (McDowd, 1986).

To assess static balance, a subtest of the Short Physical Performance Battery (SPPB) was applied (Guralnik et al., 1994; Guralnik et al., 2000). The SPPB allows an objective and valid assessment of lower extremity functioning in elderly (Guralnik et al., 1994; Guralnik et al., 2000). The balance test of the SPPB includes the following tasks: standing in (1) feet side-by-side position, (2) semi-tandem stance, and (3) full-tandem stance. Each position should be held unsupported for $10 \mathrm{~s}$. With a total score ranging from 0 ("not able to complete the tasks") to 4 ("good balance function"), the performance can be evaluated. In line with previous studies (Eggenberger et al., 2015, 2016), we extended the balance test with two additional tasks to avoid ceiling effects. The first additional task was a 20 s single-leg stance (with preferred leg) where two points were achieved for reaching $20 \mathrm{~s}$, one point for $10-20 \mathrm{~s}$ and zero points for $<10 \mathrm{~s}$. The second additional task was a single-leg stance (with preferred leg) with eyes closed where one point was assigned for every $5 \mathrm{~s}$ of successful task achievement. Three trials were conducted for each additional task whereas the best trial counted. For the extended version of the subtest, the maximum point score is unlimited with higher scores meaning better balance functioning. The total score of the extended subtest was calculated for the analysis.

Dynamic balance was assessed with the Y-Balance Test ${ }^{\mathrm{TM}}$ (YBT ${ }^{1}$, Danville, VA, United States), a valid and reliable evaluation tool (Plisky et al., 2009) often used in rather active populations (Plisky et al., 2009; Shaffer et al., 2013) and recently applied in several studies with older adults (Lee et al., 2015; Shin and An, 2015; Donath et al., 2016). The testing kit consists of a stance platform to which three PVC bars are attached in the anterior, posteromedial, and posterolateral reaching directions (the posterior bars are positioned at 135 degrees from the anterior bar with 45 degrees between both posterior bars). The participants were asked to push a target along the bars in every direction with one foot (barefoot), while standing on the other foot on the stance platform with hands on the pelvis. Thereby, the maximal reaching distance in every direction can be measured. When the personal limit was reached, participants had to return to the starting position. For the purpose of familiarization, four practice trials were performed for each foot and direction. After a short break, three testing trials were accomplished using the following order: left anterior, right anterior, left posteromedial, right posteromedial, left posterolateral, and right posterolateral. Mean values of reaching distances were calculated over all testing trials for every direction and each foot. A testing trial was classified as invalid when participants (1) failed to maintain unilateral stance on the platform, (2) failed to maintain foot contact with the target while moving the target, (3) used the target for stance support, or (4) failed to return to the starting position. Invalid testing trials were required to be repeated. For

${ }^{1}$ FunctionalMovement.com data analysis, the final score was calculated by taking the sum of the mean maximum reaching distances in each direction and for each foot (six values in total) divided by three times the sum of right and left lower limb length, and then multiplied by 100 . With this calculation, the averaged maximal reaching distance was normalized to the limb length of both legs (anterosuperior iliac spine to the most distal part of the medial malleolus) and expressed as percentage of the lower limb length. Due to safety reasons, only participants who reached $20 \mathrm{~s}$ of single-leg stance in the extended version of the SPPB balance test were eligible for the YBT.

The Senior Fitness Test (SFT), a.k.a the Fullerton Fitness Test, was used to evaluate functional fitness of the participants (Rikli and Jones, 1999). In order to assess functional lower body strength and aerobic endurance, two subtests of the SFT test battery were chosen: the $30 \mathrm{~s}$ chair rises test and the $2 \mathrm{~min}$ stepping test. In the $30 \mathrm{~s}$ chair rises test, the participants had to cross their arms in front of the chest and perform as many full "chair rises" as possible in $30 \mathrm{~s}$. A full chair rise was defined as sitting down on a chair and standing up, ending in an upright position again. The number of completed full chair rises in $30 \mathrm{~s}$ was counted. In the 2 min stepping test, the participants were asked to alternatingly step with both legs as many times as possible in 2 min while reaching a predefined individual height with the knees. This threshold height was calculated by means of the height from the floor to the middle of the thigh (midway between the iliac crest and the upper patella). For the analysis, the number of steps with the starting leg was counted during the 2 min whereas a step was valid only when the knee was reaching the required height.

\section{Cognitive Functions}

To assess cognitive functions, one computer-based test and three paper-pencil tests were used. The Test of Attentional Performance (D-TAP 2.3 VL, PSYTEST, Psychologische Testsysteme, Herzogenrath, Germany) is a computerized test battery to validly assess various attentional and executive functions (Zimmermann and Fimm, 2002). For this study, the subtest Divided attention was chosen to evaluate the capacity of dividing the attentional resources to stimuli in multiple modalities. The test was performed on a computer using an additional answer button and was preceded by a short familiarization session. Visual and acoustic signals were presented to the participants who had to react only to specific stimuli. Details about the protocol can be found elsewhere (Zimmermann and Fimm, 2002). Reaction times [ms], number of errors, and omissions were assessed.

The Trail Making Test (TMT) is a widely used, valid and reliable neuropsychological paper-pencil test to assess mental flexibility (Reitan, 1958; Corrigan and Hinkeldey, 1987; Bowie and Harvey, 2006). In the first part of the test (TMT A), participants had to connect randomly allocated, encircled numbers from 1 to 25 in ascending order as fast as possible. In the second part of the test (TMT B), the stimuli comprise encircled letters and numbers. The randomly allocated numbers and letters have to be connected in ascending numerical and alphabetical order alternatingly as fast as possible (e.g., 1 - A - 
$2-\mathrm{B}-3-\mathrm{C}-\ldots)$. In both parts, a short practice session was conducted. Time [s] for completing the tasks was recorded and errors were counted.

To assess response inhibition and interference control, the Victoria Stroop Test (VST) was used, a tool to validly and reliably measure executive functions (Strauss et al., 1991; Strauss et al., 2006; Troyer et al., 2006). The VST compromises three parts: (1) VST 1: naming the color of dots (red, blue, green, or yellow), (2) VST 2: naming the color of neutral words (e.g., words like "when" or "hardly" colored in red, blue, green, or yellow), and (3) VST 3: naming the color of "color words" printed in incongruent colors (e.g., word = red while word color = blue, etc.). In VST 3, interfering information is provided which requires interference control and response inhibition; the fast and automatic response of reading the words has to be inhibited and a more effortful color-naming response has to be produced (Bayard et al., 2011). Each part contains 24 stimuli. Performance time [s] was recorded for each task and errors were counted.

Further cognitive performance was evaluated with two subtests of the Wechsler Memory Scale-Revised (WMS-R) (Wechsler, 1987; Kent, 2013). The first subtest of the WMS-R, the digit forward task, assessed the short-term attention span and information processing speed (Wechsler, 1987; Hester et al., 2004). Participants had to remember and repeat digit sequences, which were read out loud by the tester, in the correct order. The first two sequences consisted of three digits, and afterwards, the sequence was extended with an additional digit for another two trials, and so on, until a maximum sequence length of eight digits was reached. The second subtest of the WMS-R, the digit backward task, was used to evaluate working memory capacity (Wechsler, 1987). Participants had to repeat the digit sequences in reversed order. Initially, the sequence consisted of two digits with the same extending procedure as described above, whereas the maximum sequence length was seven digits for this task. For every correct replication of a digit sequence, one point was scored, summing up to a total point score for each subtest (Peña-Casanova et al., 2009).

\section{Cortical Activity and Analysis}

Resting state EEG activity was acquired while the participants were seated comfortably for $5 \mathrm{~min}$ with eyes closed. EEG was recorded at $500 \mathrm{~Hz}$ sampling rate, using a 20-channels dryelectrodes cap (ENOBIO 20, Neuroelectrics, Barcelona, Spain) placed according to the international 10-20 system (Jasper, 1958) and referenced using the Driven-Right-Leg (DRL)/Common Mode Sense (CMS) technique (two external electrodes placed on either side of the left earlobe with an ear-clip). Before electrode placement on the forehead and earlobe, the skin was prepared with an abrasive and conductive gel $(\mathrm{H}+\mathrm{H}$ Medizinprodukte GbR, Münster, Germany).

EEG data analysis was performed using custom scripts written in MATLAB R2017b (The Mathworks, Natick, MA, United States) and using the EEGLAB 14.1.0b open source toolbox (Delorme and Makeig, 2004). EEG data was first highpass filtered [zero-phase Hamming windowed sinc FIR, cutoff frequency $(-6 \mathrm{~dB}) 0.5 \mathrm{~Hz}$, passband edge $1 \mathrm{~Hz}$, transition bandwidth $1 \mathrm{~Hz}$, order 1651] and subsequently low-pass filtered [zero-phase Hamming windowed sinc FIR, cut-off frequency (-6 dB) $45 \mathrm{~Hz}$, passband edge $40 \mathrm{~Hz}$, transition bandwidth $10 \mathrm{~Hz}$, order 167]. Further analysis was performed solely to seven parieto-occipital EEG electrodes ( $\mathrm{Pz}, \mathrm{P} 3 / 4, \mathrm{P} 7 / 8$, and $\mathrm{O} 1 / 2)$, since this cortical area is widely used to reliably detect individual alpha frequency (IAF) (Grandy et al., 2013; Corcoran et al., 2018). Channel rejection was performed using the automatic procedure supplied by the clean_rawdata EEGLAB extension by taking into account whether the correlation of a channel to a reconstruction of it based on other channels, in a given time window, was less than 0.4 , and whether a channel was flat for more than $5 \mathrm{~s}$. On average, $\sim 98 \%$ of the parieto-occipital channels in the pre-measurement EEG recordings remained for further analysis ( $\sigma: \sim 5 \%$; range: $\sim 86-100 \%$ ) and $~ 95 \%$ ( $\sigma: \sim 9 \%$; range: $\sim 71-100 \%)$ in the post-measurement EEG recordings. Artifactual data points were rejected when their amplitude was higher than $\pm 75 \mu \mathrm{V}$ within a $500 \mathrm{~ms}$ width time window as detected by the trimOutlier EEGLAB plugin. On average, 5\% of data was rejected in the pre-measurement EEG recordings ( $\sigma: \sim 11 \%$; range: $\sim 0-43 \%$ ) and $\sim 6 \%$ ( $\sigma: \sim 14 \%$; range: $\sim 0$ $17 \%)$ in the post-measurement EEG recordings. Afterwards, two IAF measures were estimated: peak alpha frequency (PAF) and center of gravity (CoG), by means of the resting IAF v1.0 open source package available from https://github.com/ corcorana/restingIAF. This allowed a fully automatic and reliable strategy to determine IAF estimates during resting state EEG recordings, of which a more detailed and extensive description can be found elsewhere (Corcoran et al., 2018; Cross et al., 2018). Briefly, one-sided channel-wise power spectral density (PSD) was first calculated in the $1-40 \mathrm{~Hz}$ frequency range by the Welch's modified periodogram method, using a 2048 sample $(\sim 4 \mathrm{~s})$ Hamming window (50\% overlap) across segments (frequency resolution $=0.244 \mathrm{~Hz}$ ) and normalized by dividing each PSD channel estimate (within the passband) by the mean spectral power. Then, each PSD estimate was smoothed using a SavitzkyGolay filter with frame length equals to 11 frequency bins and polynomial degree of five. From the smoothed PSD and within an a priori defined alpha frequency band $(7-13 \mathrm{~Hz})$, evident frequency peaks were detected and IAF estimates from spectral peaks' boundaries were computed. Using the first derivative to detect spectral peaks seemingly yields true estimates compared to simply searching for maximal values within a predefined alpha frequency band (Grandy et al., 2013). Finally, IAF estimates were computed by averaging the obtained spectral peaks estimates across channels. The minimum number of valid channels necessary to estimate IAF was set to one, given the relatively low-density parieto-occipital EEG channels used for this analysis. Additionally, spectral power within the alpha frequency band was calculated by averaging in each participant the PSD estimates of all the included channels, and then summing the obtained channels mean power across the alpha frequency band. Alpha spectral bandwidth was defined as the individual $\mathrm{PAF} \pm 2 \mathrm{~Hz}$.

\section{Other Outcome Measures}

The participants rated their current training motivation on a Visual Analog Scale ( $1=$ unmotivated lethargic smiley to 5 = motivated happy smiley) before each training session. 
The average training motivation was separately calculated for the training period in the living lab and at home. Subjective exercise intensity was reported after each training session during the whole intervention period. The participants estimated their perceived exertion on the 20-point Borg scale that ranges from 6 to 20 ( 6 = "less than very light", 20 = "more than very hard") for Tai Chi-inspired and dance exercises, respectively (Borg, 1982). To target a moderate training intensity, Borg scale ratings in the range of 12 to 14 were expected (Borg, 1982). Furthermore, objective exercise intensity was assessed by heart rate (HR) measurements during at least three training sessions performed in the living lab. A Polar M400 (Polar Electro Oy, Kempele, Finland) was used to record HR for Tai Chi-inspired and dancing training separately. The individual maximal $\mathrm{HR}\left(\mathrm{HR}_{\max }\right)$ was estimated with the formula: $\mathrm{HR}_{\max }=220-$ age. The average $\mathrm{HR}$ while training $\left(\mathrm{HR}_{\text {train }}\right)$ was compared to the individual estimated $\mathrm{HR}_{\max }$ by calculating the $\mathrm{HR}_{\text {train }}$ as percentage of the $\mathrm{HR}_{\max }$. To target a moderate training intensity, an average $\mathrm{HR}_{\text {train }}$ about $60 \%$ of $\mathrm{HR}_{\max }$ was expected (Nelson et al., 2007; Chodzko-Zajko et al., 2009).

\section{Statistical Analysis}

SPSS 23.0 for Windows (SPSS Inc, Chicago, IL, United States) was used for statistical analysis. Descriptive statistics were generated for all variables. Following a conservative approach and due to non-normality of some of the data, confirmed by both Shapiro-Wilk test and Q-Q-plots, a non-parametric testing strategy was used. Intragroup differences between pre- and postmeasurements were analyzed by Wilcoxon signed-rank test. A significance level of $\alpha=0.05$ was applied. Correlational effect sizes $(r)$, according to the following equation: $r=z / \sqrt{ }(n 1+n 2)$ with $\mathrm{n}=$ sample size, $\mathrm{n} 1=\mathrm{n}$ at pre-measurement and $\mathrm{n} 2=\mathrm{n}$ at post-measurement, were calculated in MS Office Excel (version 2016) and reported according to Cohen (Cohen, 1988): an effect size of $r=0.10$ indicates a small effect, $r=0.30$ a medium effect, and $r \geq 0.50$ a large effect. For pre- and post-measurement comparisons, drop-outs were excluded from analysis (perprotocol analysis). The analysis does not consider intentionto-treat analysis because of a clear description of the drop-out reasons (Moher et al., 2010). Moreover, only participants who reached $70 \%$ of the maximal possible training sessions were included in the pre-post-comparison.

\section{RESULTS}

Twenty-one participants signed informed consent and were included in the study. Fifteen participants completed the 8-week training intervention. Six participants prematurely terminated study participation. The study flow chart is presented in Figure 2. Table 1 summarizes the demographic characteristics and screening measures of the drop-outs and the remaining participants separately. The drop-outs were comparable to the rest of the participants regarding their characteristics except of age $(U=75.50, p=0.017)$ and self-evaluated balance at baseline $\left[\chi^{2}(\mathrm{df})=9.64(3), p=0.022\right]$.

\section{Primary Outcome: Feasibility and Usability}

The adherence rate over the whole intervention period was 91.1\% $(n=15)$. For the training period in the living lab, the attendance rate reached $91.7 \%$ whereas $91.0 \%$ of all training sessions were attended during the training period at home. Both attendance rates were above the predefined $70 \%$ threshold rate, which was considered as acceptable adherence. Ten participants completed all planned 24 training sessions. Reasons for not attending training sessions were: holidays, being busy, work, family affairs, and temporary health issues (e.g., catching a cold). After the 8-week training intervention, the attrition rate of $28.6 \%$ (six drop-outs) was higher than the predefined expectable drop-out rate of $10 \%$. Nevertheless, the sample size required for gaining valuable preliminary information remained well above the recommended 12 individuals. Three drop-out reasons were associated with technical problems and malfunctions of the training systems (software problems with "system crashes," unstable IMU connections, inappropriate movement evaluation), which led to a decreased training motivation. In the other three drop-outs, the reasons for terminating the study were related to either personal or health issues (injury, sudden illness, family affairs).

During the intervention period, none of the 15 participants suffered any adverse events related to the study intervention, especially no falls or injuries during measurements or training. Moreover, no fear of falling during exergame training was reported. However, one participant reported to have experienced a "critical moment" where she almost fell because of swaying during a strength exercise in a lunge position. Light dizziness during training was reported by three participants (related to body rotations during dancing). Slight pain during training was stated by three participants whereas in one case, the pain was related to a foot injury which happened unrelated to the training. The other two participants reported slight knee and/or back pain.

The SUS score was $70.6 \pm 19.8(n=17)$ showing acceptable usability (a predefined 70-point score was considered acceptable for usability). In the additional question, asking the participants about their general opinion of the Active@Home exergame, the mean score was $3.2 \pm 1.0$ (on a scale from $0=$ "I don't like it" to $4=$ "I like it a lot"). Figure 3 presents the results of the GEQ $(n=17)$, indicating positive game experience based on the above-average score of positive affect $(2.3 \pm 1.0)$ and the low scores of negative affect $(0.3 \pm 0.5)$ and tension $(0.8 \pm 0.7)$. The ratings for competence $(2.2 \pm 0.9)$ and immersion $(2.0 \pm 0.7)$ were around average whereas the scores of challenge $(1.6 \pm 0.6)$ and flow $(1.3 \pm 0.8)$ were slightly lower. Table 2 summarizes the main feedback of the participants and the observations of the supervisors during the training in the living lab setting.

\section{Secondary Outcome: Effects of the Intervention}

Table 3 summarizes median and variability values for the measures of secondary outcome.

For gait analysis, the minimal toe clearance under single-task walking condition increased significantly $(z=-2.158, p=0.030$, 


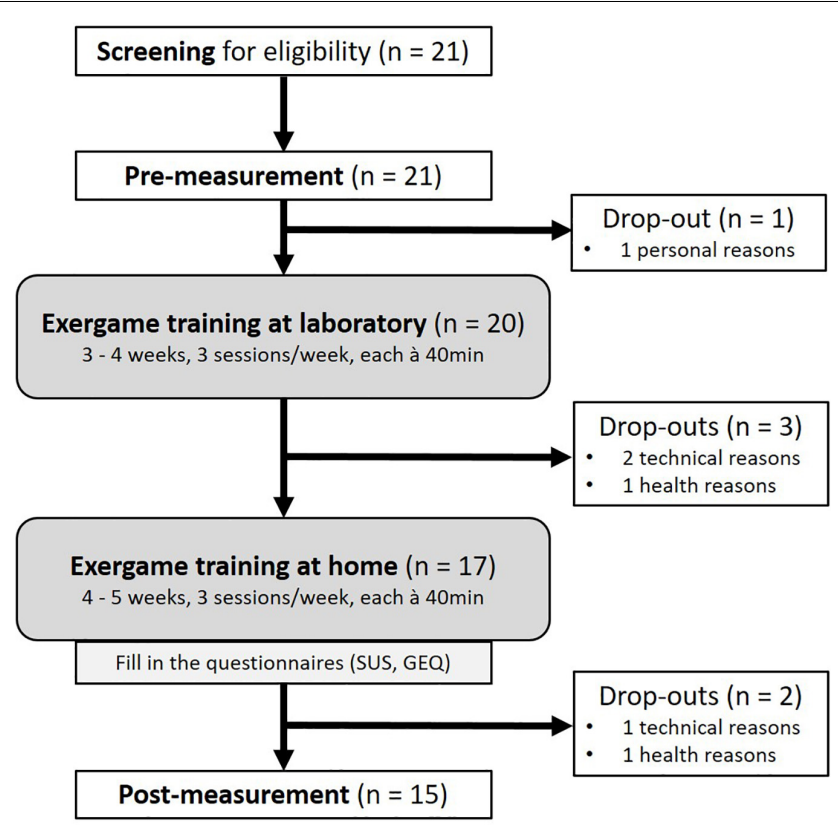

FIGURE 2 | Study flow chart. Screening of participants for eligibility included an assessment of cognitive and health state. Physical and cognitive functions as well as brain activity were measured at pre- and post-measurement. Assessments and the first half of the intervention were performed at the living lab of ETH Hönggerberg (Zurich, Switzerland), the second half at participants' home. Questionnaires assessing usability and game experience were filled in after the training period. Technical drop-out reasons included software problems, unstable IMU connections and inappropriate movement evaluation. Other drop-out reasons were related to injuries, sudden illness and family affairs. SUS, System Usability Scale; GEQ, Game Experience Questionnaire.

$r=0.39)$ comparing pre- and post-measurement. Considering the balance assessments, nine participants reached $20 \mathrm{~s}$ or more for the single-leg stance and, thus, were eligible for the YBT test. A medium to large effect size $(z=-1.836, p=0.074, r=0.43)$ was evident for these individuals with a tendency of higher dynamic balance scores after the intervention. In the digit forward task of the WMS-R, a significant increase in the performance score was found $(z=-2.859, p=0.002, r=0.52)$ comparing pre- and post-measurement. Furthermore, reaction time in the first task of the VST (VST 1) was significantly faster $(z=-2.727, p=0.004$, $r=0.50)$ after the training intervention. No significant changes in resting state EEG were found for the pre-post-comparison. However, a medium to large effect size was evident for the alpha spectral power $(z=-1.955, p=0.055, r=0.44)$ with a tendency of increased spectral power in the individual alpha frequency band after training.

\section{Other Outcome Results}

Participants rated their training motivation on average with $4.2 \pm 0.7(n=20)$. The motivation for the training in the living lab was $4.2 \pm 0.6(n=20)$ and the motivation for the home-based training was $4.1 \pm 0.8(n=17)$. The average rating of participants' perceived exertion for Tai Chi-inspired exercises was $11.9 \pm 1.5$ $(n=20)$ and for dance exercises $11.0 \pm 1.5(n=20)$. Both ratings reflect an intensity on the 20-point Borg scale that corresponds from "fairly light" (11) to "somewhat hard" (13). The average HR during Tai Chi-inspired training was $60 \pm 10 \%$ of the individual maximal $\mathrm{HR}$, the average $\mathrm{HR}$ during dancing training was also $61 \pm 11 \%$ of the $\mathrm{HR}_{\max }$.

\section{DISCUSSION}

The primary aim of this study was to evaluate the feasibility and usability of an in-home exergame training for older adults. Furthermore, potential training-related adaptations, both in physical and cognitive aspects of functioning, were explored. In summary, the study results showed a general feasible and well-accepted exergame that indicates to endorse measurable training effects in certain physical and cognitive functions.

\section{Feasibility and Usability}

The conclusion of a feasible training system is based on the high adherence rate of over $90 \%$. Interestingly, the adherence rates for the training periods in the living lab and at home were almost the same $(91.7 \%$ and $91.0 \%$, respectively). Therefore, the presence of a supervisor during the living lab training sessions might not have promoted the training adherence compared to the in-home setting. Although, a widespread opinion is that social interaction increases training motivation in older adults (Smith et al., 2017). The high adherence rate found in this study is in line with the result of the first exploratory study testing the newly developed exergame in a laboratory setting. Furthermore, previous studies showed high training attendance in older adults using exergames (De Bruin et al., 2011; Wuest et al., 2014). Exergame interventions often showed higher adherence compared to standard fall prevention exercises (Sjösten et al., 2007; De Bruin et al., 2011; Wuest et al., 2014). One reason might be the high motivational potential 
TABLE 1 | Demographic characteristics of participants and screening values.

\begin{tabular}{|c|c|c|}
\hline Participant characteristics & $n=21$ & $n=6$ (drop-outs) \\
\hline Age in years & $74.4 \pm 7.0(65-92)$ & $80.2 \pm 7.1(70-92)$ \\
\hline Weight $[\mathrm{kg}]$ & $73.2 \pm 19.8(42-120)$ & $66.0 \pm 20.0(42-95)$ \\
\hline Height [cm] & $168.0 \pm 9.4(155-187)$ & $168.0 \pm 12.4(155-187)$ \\
\hline Education in years & $14.1 \pm 4.2(4-20)$ & $13.8 \pm 6.1(4-20)$ \\
\hline MMSE Score & $29.0 \pm 1.6(24-30)$ & $27.8 \pm 2.6(24-30)$ \\
\hline Female [n,\%] & $11(52.4)$ & $2(33.3)$ \\
\hline \multicolumn{3}{|l|}{ Fear of falling $[n, \%]$} \\
\hline Never & $17(81.0)$ & $6(100.0)$ \\
\hline Sometimes & $3(14.3)$ & $0(0.0)$ \\
\hline Often & $1(4.8)$ & $0(0.0)$ \\
\hline Always & $0(0.0)$ & $0(0.0)$ \\
\hline \multicolumn{3}{|l|}{$\begin{array}{l}\text { Number of falls during last } \\
\text { month }^{+}[n, \%]\end{array}$} \\
\hline Never & $18(85.7)$ & $6(100.0)$ \\
\hline Once & $2(9.5)$ & $0(0.0)$ \\
\hline More than once & $1(4.8)$ & $0(0.0)$ \\
\hline \multicolumn{3}{|l|}{$\begin{array}{l}\text { Self-evaluation of health } \\
\text { state }[n, \%]\end{array}$} \\
\hline Very good & $5(23.8)$ & $1(16.7)$ \\
\hline Good & $12(57.1)$ & $2(33.3)$ \\
\hline Medium & $4(19.0)$ & $3(50.0)$ \\
\hline Bad & $0(0.0)$ & $0(0.0)$ \\
\hline \multicolumn{3}{|l|}{$\begin{array}{l}\text { Self-evaluation of balance } \\
{[n, \%]}\end{array}$} \\
\hline Very good & $3(14.3)$ & $3(50.0)$ \\
\hline Good & $9(42.9)$ & $1(16.7)$ \\
\hline Medium & 7 (33.3) & $2(33.3)$ \\
\hline $\mathrm{Bad}$ & $2(9.5)$ & $0(0.0)$ \\
\hline \multicolumn{3}{|l|}{$\begin{array}{l}\text { Self-evaluation of muscle } \\
\text { strength [n,\%] }\end{array}$} \\
\hline Very good & $2(9.5)$ & $1(16.7)$ \\
\hline Good & $12(57.1)$ & $4(66.7)$ \\
\hline Medium & 7 (33.3) & $1(16.7)$ \\
\hline Bad & $0(0.0)$ & $0(0.0)$ \\
\hline
\end{tabular}

Data are mean values \pm standard deviations (ranges) or number of participants per category (absolute and relative frequencies). MMSE, Mini Mental State Examination. + Self-stated.

and playfulness of exergames leading to a captivating and entertaining training (Vaziri et al., 2016; Chesham et al., 2017; Valenzuela et al., 2018). Accordingly, the motivation ratings in this study were equally high for the training in the living lab and at home. In general, high motivation seems to be crucial for the success of training interventions as motivation might lead to a high training compliance and, therefore, training related benefits can reach their full potential (van Het Reve et al., 2014). On the other hand, the attrition rate in this study was higher than the predefined rate (Nyman and Victor, 2012). Even after subtracting the drop-outs resulting from reasons not related to the training system itself (e.g., due to health issues), attrition was still higher than the predefined rate. Although, the drop-out rate is about $10 \%$ on average in exergame studies (Skjæret et al., 2016), it can reach up to $40 \%$ in individual studies (Larsen et al., 2013). Exergames might be more prone to technical issues leading, in some cases, to higher drop-out rates. Interestingly, the drop-outs were not related to safety concerns of participants (e.g., fear of falling during training) or even injuries related to exercising. In general, participants reported no safety issues beside of isolated cases of light insecurities or dizziness. It has to be considered, that the Active@Home exergame was developed for healthy older adults living independently at home. Exercises might have to be adapted if the exergame should target clinical populations.

Despite the rather high attrition rate, the usability of the exergame was rated with an acceptable score (SUS score of $70.6 / 100$ ) and a general positive feedback was given by the participants. They were satisfied with the game story, the virtual instructor, and the training content including diverse and challenging exercises and cognitive stimulation. Comparing to existing literature, exergames in general have been shown to be well accepted and usable for healthy older adults (Gerling and Mandryk, 2014; Nawaz et al., 2016; Vaziri et al., 2016). Additionally to the participants' feedback, supervisors' observation during the training period in the living lab gave further insights in the general positive user experiences e.g., easy usable system set up and simple attachment of the IMUs. However, some discrepancies were evident comparing the supervisors' observations and the participants' positive feedback. Despite the high rated usability, difficulties in using the technology-based system (e.g., navigating through the game) have been observed and help was occasionally requested by participants. Previous experiences with technology might have been beneficial as for example the navigation by the hand IMU could be partly related to previous experiences with other navigation systems, e.g., the computer mouse. The most threatening aspect for the usability of the Active@Home exergame was related to technical malfunctions of the system. Participants often were frustrated and disappointed when the IMU connections were unstable or movement detection was incorrect. Therefore, a technology-based training system with flawless functionality and age-appropriate design may be crucial considering the potentially restricted technology knowledge of older adults and the independent use of the training system at home (Brach et al., 2012; Bleakley et al., 2015; Vaziri et al., 2016).

The emotional reactions evident in the observations were also reflected in the results of a questionnaire assessing emotions and game experience (GEQ). Compared to other exergame studies using this questionnaire (Gerling et al., 2011; Liukkonen et al., 2015; Merriman et al., 2018), positive affect was rated slightly lower whereas tension was rated slightly higher in this study. Nevertheless, the emotional experience during training with the Active@Home exergame can be interpreted as general positive considering ratings of positive emotions above average (e.g., feeling captivated and pleased) and a low rating of negative affect (e.g., feeling tensed and annoyed). Emotions related to challenge and flow were rated rather low which might be explained by inappropriate training load and by technical problems interrupting the game flow. In sum, the Active@Home exergame provided mainly positive emotional experiences which 


\section{Game Experience Questionnaire}

\section{4}

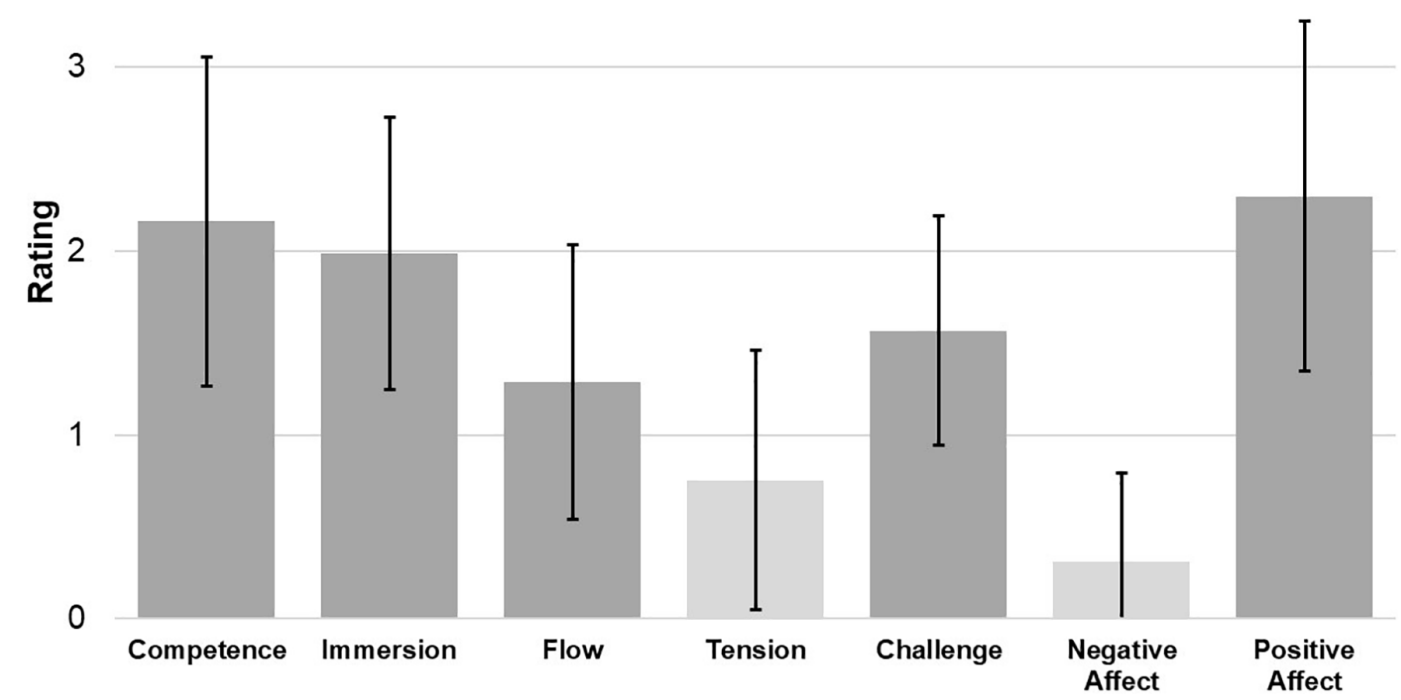

FIGURE 3 | Primary outcome result of game experience. Data shown in the figure are means with standard deviations in each emotional category of the GEQ $(n=17)$. The two light-gray categories (tension, negative affect) have to be evaluated reversely which means a lower score is favorable. The GEQ ranges from $0=$ "not at all" to 4 = "extremly".

TABLE 2 | Primary outcome results: summary of usability protocol with supervisors' observations and participants' feedback.

\begin{tabular}{|c|c|c|}
\hline Criteria & Positive aspects & Negative aspects \\
\hline IMUs & $\begin{array}{l}\text { - Comfortable to wear } \\
\text { - Easy to attach (slap band) to wrists/ankles } \\
\text { - Slap bands are easy to clean (e.g., from sweat) } \\
\text { - Feasible navigation with the right hand IMU ("hand mouse") } \\
\text { - Battery can be easily charged }\end{array}$ & $\begin{array}{l}\text { - Instable IMU connection (via Bluetooth) } \\
\text { - Activation of the "hand mouse" in inappropriate situations (e.g., } \\
\text { during dancing) } \\
\text { - Suboptimal navigation for left-hander } \\
\text { - Technical malfunctions of IMUs }\end{array}$ \\
\hline Design & $\begin{array}{l}\text { - Appealing game design } \\
\text { - Pleasant and diverse music } \\
\text { - Cute and funny cartoon-based instructor }\end{array}$ & $\begin{array}{l}\text { - Virtual instructor could be more motivating (gestures as "thumps } \\
\text { up", comments etc.) }\end{array}$ \\
\hline Training principles & $\begin{array}{l}\text { - Real-time feedback while exercising (auditory and visual, positive } \\
\text { and negative) } \\
\text { - Performance score as feedback after exercising is motivating } \\
\text { - High variability of exercises and levels }\end{array}$ & $\begin{array}{l}\text { - Auditory feedback (sounds for "right" and "wrong") could be } \\
\text { louder in relation to background music } \\
\text { - Feedback not always traceable } \\
\text { - Partly inaccurate evaluation of movements }\end{array}$ \\
\hline Emotions & $\begin{array}{l}\text { - Motivation and fun (despite of technical malfunctions) } \\
\text { - Curiosity about what is coming in the next levels } \\
\text { - Happiness when seeing progress or achieving more performance } \\
\text { points }\end{array}$ & $\begin{array}{l}\text { - Frustration and displeasure because of technical issues and } \\
\text { inaccurate evaluation of movements } \\
\text { - Impatience with navigation }\end{array}$ \\
\hline
\end{tabular}

IMU, inertial measurement unit. 
TABLE 3 | Secondary outcome results.

\begin{tabular}{|c|c|c|c|c|c|c|}
\hline Secondary Outcomes & Assessed by & Pre & Post & $z$ & $p$ & $r$ \\
\hline \multirow[t]{24}{*}{ Physical functions } & \multicolumn{6}{|l|}{ Gait analysis } \\
\hline & \multicolumn{6}{|l|}{ Speed $[\mathrm{m} / \mathrm{s}]$} \\
\hline & ST walking & $1.41(1.32 ; 1.49)$ & $1.43(1.23 ; 1.55)$ & -0.454 & 0.679 & 0.08 \\
\hline & DT walking & $1.23(1.13 ; 1.42)$ & $1.34(1.12 ; 1.44)$ & -1.590 & 0.121 & 0.29 \\
\hline & DT costs [\%] & $9.4(6.5 ; 18.3)$ & $10.1(2.5 ; 17.2)$ & -1.477 & 0.151 & 0.27 \\
\hline & \multicolumn{6}{|l|}{ Cadence [steps/min] } \\
\hline & ST walking & $119.4(114.8 ; 120.7)$ & 118.9 (111.3; 123.3) & -0.909 & 0.389 & 0.17 \\
\hline & DT walking & $112.9(103.1 ; 114.8)$ & $113.3(104.7 ; 117.3)$ & -1.704 & 0.095 & 0.31 \\
\hline & DT costs [\%] & $6.8(3.6 ; 12.1)$ & $5.1(3.2 ; 12.0)$ & -1.533 & 0.135 & 0.28 \\
\hline & \multicolumn{6}{|l|}{ Stride length [m] } \\
\hline & ST walking & $1.40(1.35 ; 1.51)$ & $1.41(1.33 ; 1.47)$ & -0.341 & 0.762 & 0.06 \\
\hline & DT walking & $1.30(1.25 ; 1.52)$ & $1.40(1.25 ; 1.51)$ & -1.022 & 0.330 & 0.19 \\
\hline & DT costs [\%] & $4.7(0.1 ; 6.9)$ & $3.1(-1.6 ; 5.9)$ & -0.795 & 0.454 & 0.15 \\
\hline & \multicolumn{6}{|l|}{ Minimal toe clearance $[\mathrm{cm}]$} \\
\hline & ST walking & $1.9(1.5 ; 2.4)$ & $2.3(1.9 ; 3.0)$ & -2.158 & $0.030 *$ & 0.39 \\
\hline & DT walking & $2.0(1.5 ; 2.7)$ & $2.4(1.5 ; 3.1)$ & -1.306 & 0.208 & 0.24 \\
\hline & DT costs [\%] & $-4.5(-36.3 ; 2.4)$ & $6.2(-10.5 ; 16.4)$ & -0.852 & 0.421 & 0.16 \\
\hline & \multicolumn{6}{|c|}{ Extended Balance Test of SPPB } \\
\hline & Balance score & $7(6 ; 7)$ & $7(6 ; 7)$ & -0.857 & 0.488 & 0.16 \\
\hline & \multicolumn{6}{|l|}{ Senior Fitness Test (SFT) } \\
\hline & $30 \mathrm{~s}$ chair rises test & $15(13 ; 20)$ & $18(15 ; 22)$ & -1.603 & 0.110 & 0.29 \\
\hline & 2 min stepping test & $94(74 ; 114)$ & $100(79 ; 113)$ & -1.137 & 0.270 & 0.21 \\
\hline & \multicolumn{6}{|l|}{ Y-Balance Test (YBT) } \\
\hline & YBT score $[\%]^{+}$ & $76.8(73.7 ; 85.2)$ & $87.1(73.9 ; 91.1)$ & -1.836 & 0.074 & 0.43 \\
\hline \multirow[t]{25}{*}{ Cognitive functions } & \multicolumn{6}{|c|}{ Divided Attention Test of TAP } \\
\hline & RT auditory [ms] & $624(577 ; 675)$ & $648(617 ; 691)$ & -0.057 & 0.966 & 0.01 \\
\hline & $\mathrm{RT}$ visual [ms] & 834 (798; 980) & $892(813 ; 1012)$ & -0.454 & 0.679 & 0.08 \\
\hline & Errors & $3(1 ; 5)$ & $3(1 ; 5)$ & -0.466 & 0.730 & 0.09 \\
\hline & Omissions & $2(1 ; 3)$ & $2(0 ; 3)$ & -0.051 & 0.953 & 0.01 \\
\hline & \multicolumn{6}{|l|}{ Trial Making Test (TMT) } \\
\hline & \multicolumn{6}{|l|}{ TMT A } \\
\hline & Time $[\mathrm{s}]$ & $38.3(35.1 ; 46.5)$ & $33.6(31.2 ; 50.0)$ & -0.625 & 0.561 & 0.11 \\
\hline & Errors & $0(0 ; 0)$ & $0(0 ; 0)$ & -1.000 & 1.000 & 0.18 \\
\hline & \multicolumn{6}{|l|}{ TMT B } \\
\hline & Time $[s]$ & $85.9(72.0 ; 107.8)$ & $85.5(70.5 ; 109.0)$ & -0.511 & 0.639 & 0.09 \\
\hline & Errors & $0(0 ; 2)$ & $1(0 ; 2)$ & -0.520 & 0.656 & 0.09 \\
\hline & \multicolumn{6}{|c|}{ Wechsler Memory Scale-Revised (WMS-R) } \\
\hline & Digit forward task & $6(6 ; 8)$ & $8(6 ; 9)$ & -2.859 & $0.002^{*}$ & 0.52 \\
\hline & Digit backward task & $5(5 ; 6)$ & $6(6 ; 7)$ & -0.890 & 0.434 & 0.16 \\
\hline & \multicolumn{6}{|l|}{ Victoria Stroop Test (VST) } \\
\hline & \multicolumn{6}{|l|}{ VST 1} \\
\hline & Time $[s]$ & $13.2(12.4 ; 15.2)$ & $12.5(11.4 ; 14.5)$ & -2.727 & $0.004^{*}$ & 0.50 \\
\hline & Errors & $0(0 ; 0)$ & $0(0 ; 0)$ & -0.707 & 0.750 & 0.13 \\
\hline & \multicolumn{6}{|l|}{ VST 2} \\
\hline & Time [s] & $16.5(14.1 ; 19.2)$ & $15.2(14.6 ; 16.1)$ & -1.789 & 0.075 & 0.33 \\
\hline & Errors & $0(0 ; 0)$ & $0(0 ; 0)$ & -1.414 & 0.500 & 0.26 \\
\hline & \multicolumn{6}{|l|}{ VST 3} \\
\hline & Time $[\mathrm{s}]$ & $25.0(22.0 ; 29.0)$ & $22.8(19.0 ; 27.2)$ & -1.533 & 0.135 & 0.28 \\
\hline & Errors & $0(0 ; 1)$ & $0(0 ; 1)$ & -1.023 & 0.328 & 0.19 \\
\hline Cortical activity & Resting state EEG & & & & & \\
\hline & Peak alpha frequency [Hz] & $9.3(8.7 ; 9.5)$ & $9.7(9.0 ; 9.8)$ & -1.104 & 0.375 & 0.25 \\
\hline & Center of gravity $[\mathrm{Hz}]$ & $8.8(8.5 ; 9.5)$ & $9.2(8.8 ; 9.5)$ & -1.682 & 0.105 & 0.36 \\
\hline & Alpha spectral power $\left[\mu \mathrm{V}^{2}\right]$ & $23.4(20.1 ; 39.2)$ & $27.8(19.8 ; 63.9)$ & -1.955 & 0.055 & 0.44 \\
\hline
\end{tabular}

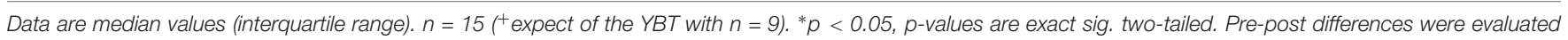

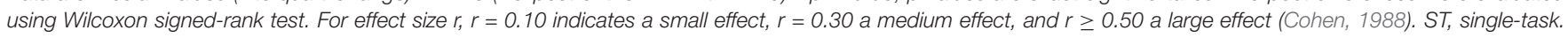
DT, dual-task. DT costs are calculated as (ST-DT)/ST × 100. SPPB, Short Physical Performance Battery. TAP, Test of Attentional Performance. RT, reaction time. 
might be linked to the high training motivation as well as the high training adherence.

\section{Effects of the Intervention}

Our study results showed a significant improvement in minimal toe clearance under single-task walking leading to higher "foot lifting" after training which might be related to a decreased risk of tripping and falling in older adults (Barrett et al., 2010). Improvements in gait parameters after exergame training have been found in several previous studies, mainly under dual-task walking (Pichierri et al., 2012; Schättin et al., 2016; Wang et al., 2017). These effects on multitasking capabilities are discussed to be due to the characteristics of exergame training targeting the physical-cognitive interplay (Segev-Jacubovski et al., 2011; Beurskens and Bock, 2012). Even though the Active@Home exergame included training of physical and cognitive functions, no significant improvements in gait parameters under dualtask walking have been found in this study. Regarding the other assessed physical functions, the statistical analysis did not reveal any significant changes after the training intervention. Nevertheless, a medium to large effect size was found for dynamic balance assessed by the YBT despite the small sample size due to restricted participant eligibility. In contrast, measures of static balance assessed by the SPPB showed no improvement which is in line with the results of the first Active@Home exergame study. We might conclude that the Active@Home exergame has a stronger focus on training of dynamic balance or that the SPPB is not sensitive enough to assess balance differences in an upper range of static balance performance. Moreover, optimal training load in a moderate to vigorous exercise intensity might be questioned considering the subjective ratings of perceived exertion during trainings (Borg scale ratings) and objectively assessed training intensity (heart rate measurements) (Nelson et al., 2007; Chodzko-Zajko et al., 2009). The suboptimal training load and the rather short training period might have restricted the training impact on additional physical outcomes. Therefore, further studies should focus on ensuring optimal training intensity as well as extended training duration.

In the cognitive tests, study results showed a significant change in the short-term attentional span (i.e., digit forward task of WMS-R), whereas an improvement in general attentional focus and information processing speed might be assumed. Accordingly, information processing speed in another task (i.e., VST 1) significantly improved after the training intervention resulting in faster reaction times. These positive effects might be attributed to the cognitive training components and the physicalcognitive interaction required in the exergame training. Similarly, recent studies showed significant enhancement of cognitive abilities including information processing speed after exergame training in older adults (Maillot et al., 2012; Schoene et al., 2013; Ogawa et al., 2016; Stanmore et al., 2017). Interestingly, a cognitive training alone (e.g., computer-based training) without elements of physical activity seems to have only small or none effects on cognitive functions (Lampit et al., 2015) leading to the suggestion that exergames have a greater impact on cognition than sole computer-based training. Nevertheless, no significant changes were evident for executive functions as mental flexibility and inhibition. As mentioned before, the rather short intervention period might have also limited the training effects on cognitive functions.

Our study results of the neuronal activity measurements during resting state EEG showed a medium to large effect size for alpha spectral power with a tendency to a power increase after the training intervention. A variety of studies reported agerelated changes in cortical oscillatory activity (Klimesch, 1999), often summarized as age-related "slowing" and described as an EEG power increase in the slow frequency ranges $(<7 \mathrm{~Hz})$ and a decrease in higher frequencies $(>7 \mathrm{~Hz})$, especially in posterior brain regions (Rossini et al., 2007; Babiloni et al., 2016). Moreover, the individual alpha frequency peak is known to decrease in the later part of lifespan (Klimesch, 1999). A positive correlation between EEG power within the alpha frequency band and global cognitive status in healthy as well as in impaired older adults is considered (Klimesch, 1999; Vecchio et al., 2013). Our study results support the suggestion that the age-related "slowing" in cortical oscillatory activity can be counteracted with physical and cognitive training (Babiloni et al., 2016). However, we found no significant changes in measurements of the IAF.

\section{Limitations}

As this is a pilot study with primary focus on feasibility and usability outcomes, secondary results have to be interpreted with caution (Arain et al., 2010; Thabane et al., 2010). The small sample size might have limited the detection of significant training effects comparing pre- and post-measurements. Nevertheless, pilot studies are especially performed to generate preliminary data to allow system and protocol development for future studies. Based on the "rule of 12", we can be confident about our values (Moore et al., 2011). Moreover, the lack of a control group might have limited further explorative analyses of training effects comparing pre- and post-measurements. As next step, a randomized controlled trial should be conducted to carefully evaluate the effectiveness of the Active@Home exergame. Moreover, in future studies, the training period should be extended to increase the potential for training improvements.

\section{CONCLUSION}

Our study results showed a general high feasibility and usability of the adapted Active@Home exergame and an overall positive emotional game experience in a living lab as well as in a home-based setting. These results lead to the conclusion that the presence of a supervisor might not be a crucial factor regarding training compliance and motivation. Furthermore, this study demonstrated that older adults were able to use the exergame in an in-home setting. However, simplicity and flawless technical functionality of the training system should be a mandatory development consideration. Moreover, a rather high variability in participants' general feedback about the exergame training was present reflecting the individual experiences and preferences. Thus, we might conclude that the Active@Home exergame is a general feasible and usable in-home training satisfying the needs and requirements of at least a part 
of the older population. Additionally, results of physical and cognitive testing indicate that the exergame might have positive influence on crucial functions in older adults. However, the efficacy has to be evaluated in a future randomized controlled trial.

\section{DATA AVAILABILITY STATEMENT}

The raw data supporting the conclusions of this manuscript will be made available by the authors, without undue reservation, to any qualified researcher upon request.

\section{ETHICS STATEMENT}

This study was carried out in accordance with the recommendations of the ETH Zürich Ethics Committee (Zurich, Switzerland). All subjects gave written informed consent in accordance with the Declaration of Helsinki. The protocol was approved by the ETH Zürich Ethics Committee (EK 2018-N-07).

\section{AUTHOR CONTRIBUTIONS}

MA and MT developed the research question under the lead of EB. MA and MT established the concept and design while AS and EB acted as methodological council. MA and MT conducted

\section{REFERENCES}

Amboni, M., Barone, P., and Hausdorff, J. M. (2013). Cognitive contributions to gait and falls: evidence and implications. Mov. Disord. 28, 1520-1533. doi: $10.1002 /$ mds. 25674

American Geriatrics Society, British Geriatrics Society, and American Academy of Orthopaedic Surgeons Panel on Falls Prevention (2001). Guideline for the prevention of falls in older persons American Geriatrics Society, British Geriatrics Society, and American Academy of Orthopaedic Surgeons Panel on Falls Prevention. J. Am. Geriatr. Soc. 49, 664-672. doi: 10.1046/j.1532-5415. 2001.49115.x

Aminian, K., Robert, P., Buchser, E., Rutschmann, B., Hayoz, D., and Depairon, M. (1999). Physical activity monitoring based on accelerometry: validation and comparison with video observation. Med. Biol. Eng. Comput. 37, 304-308. doi: 10.1007/bf02513304

Arain, M., Campbell, M. J., Cooper, C. L., and Lancaster, G. A. (2010). What is a pilot or feasibility study? A review of current practice and editorial policy. BMC Med. Res. Methodol. 10:67. doi: 10.1186/1471-2288-10-67

Babiloni, C., Lizio, R., Marzano, N., Capotosto, P., Soricelli, A., Triggiani, A. I., et al. (2016). Brain neural synchronization and functional coupling in Alzheimer's disease as revealed by resting state EEG rhythms. Int. J. Psychophysiol. 103, 88-102. doi: 10.1016/j.ijpsycho.2015.02.008

Bamidis, P., Vivas, A., Styliadis, C., Frantzidis, C., Klados, M., Schlee, W., et al. (2014). A review of physical and cognitive interventions in aging. Neurosci. Biobehav. Rev. 44, 206-220.

Bangor, A., Kortum, P., and Miller, J. (2009). Determining what individual SUS scores mean: adding an adjective rating scale. J. Usabil. Stud. 4, 114-123.

Barrett, R., Mills, P., and Begg, R. (2010). A systematic review of the effect of ageing and falls history on minimum foot clearance characteristics during level walking. Gait Posture 32, 429-435. doi: 10.1016/j.gaitpost.2010.07.010 data acquisition, analysis and interpretation of the results with edition and improvement by AS and EB. FG performed EEG data analysis and interpretation of the EEG results, which was edited and improved by MA, AS, and EB. MA and MT produced a first version of the manuscript. AS, FG, and EB substantially revised the manuscript to bring it to its current version. All authors have read and approved the final manuscript.

\section{FUNDING}

This work was partially supported by the AAL Active@Home project (www.aal-europe.eu/projects/activehome/), funded by the European Commission and co-funded by the Swiss Confederation represented by the State Secretariat for Education, Research and Innovation (SERI; agreement number 1315000595).

\section{ACKNOWLEDGMENTS}

We would like to thank Jennifer Post for supporting testing and training. Furthermore, we would like to thank all participants for their kindness and patience during the testing and training sessions. We would like to thank also all the national and international partners involved in the Active@Home project and exergame development, with very special thanks to Vânia Guimarães.

Bayard, S., Erkes, J., and Moroni, C. (2011). Victoria Stroop Test: normative data in a sample group of older people and the study of their clinical applications in the assessment of inhibition in Alzheimer's disease. Arch. Clin. Neuropsychol. 26, 653-661. doi: 10.1093/arclin/acr053

Beurskens, R., and Bock, O. (2012). Age-related deficits of dual-task walking: a review. Neural Plast. 2012:131608. doi: 10.1155/2012/131608

Bleakley, C. M., Charles, D., Porter-Armstrong, A., McNeill, M. D., McDonough, S. M., and McCormack, B. (2015). Gaming for health a systematic review of the physical and cognitive effects of interactive computer games in older adults. J. Appl. Gerontol. 34, 166-189. doi: 10.1177/0733464812470747

Borg, G. A. (1982). Psychophysical bases of perceived exertion. Med. Sci. Sports Exerc. 14, 377-381.

Bowie, C. R., and Harvey, P. D. (2006). Administration and interpretation of the Trail Making Test. Nat. Protoc. 1, 2277-2281. doi: 10.1038/nprot.2006.390

Brach, M., Hauer, K., Rotter, L., Werres, C., Korn, O., Konrad, R., et al. (2012). Modern principles of training in exergames for sedentary seniors: requirements and approaches for sport and exercise sciences. Int. J. Comput. Sci. Sport 11, 86-99.

Brooke, J. (1996). SUS-A quick and dirty usability scale. Usabil. Eval. Ind. 189, 4-7.

Brox, E., Konstantinidis, S. T., and Evertsen, G. (2017). User-centered design of serious games for older adults following 3 years of experience with exergames for seniors: a study design. JMIR Serious Games 5:e2. doi: 10.2196/games.6254

Campbell, M., Fitzpatrick, R., Haines, A., Kinmonth, A. L., Sandercock, P., Spiegelhalter, D., et al. (2000). Framework for design and evaluation of complex interventions to improve health. Br. Med. J. 321, 694-696. doi: 10.1136/bmj.321. 7262.694

Chesham, A., Wyss, P., Müri, R. M., Mosimann, U. P., and Nef, T. (2017). What older people like to play: genre preferences and acceptance of casual games. JMIR Serious Games 5:e8. doi: 10.2196/games.7025

Chodzko-Zajko, W. J. (2014). Exercise and physical activity for older adults. Kinesiol. Rev. 3, 101-106. 
Chodzko-Zajko, W. J., Proctor, D. N., Singh, M. A. F., Minson, C. T., Nigg, C. R., Salem, G. J., et al. (2009). Exercise and physical activity for older adults. Med. Sci. Sports Exerc. 41, 1510-1530.

Choi, S. D., Guo, L., Kang, D., and Xiong, S. (2017). Exergame technology and interactive interventions for elderly fall prevention: a systematic literature review. Appl. Ergon. 65, 570-581. doi: 10.1016/j.apergo.2016.10.013

Cohen, J. (1988). Statistical Analysis for the Behavioral Sciences. Hillsdale, NJ: Lawrance Erlbaum.

Corcoran, A. W., Alday, P. M., Schlesewsky, M., and Bornkessel-Schlesewsky, I. (2018). Toward a reliable, automated method of individual alpha frequency (IAF) quantification. Psychophysiology 55:e13064. doi: 10.1111/psyp.13064

Corrigan, J. D., and Hinkeldey, N. S. (1987). Relationships between parts A and B of the Trail Making Test. J. Clin. Psychol. 43, 402-409. doi: 10.1002/10974679(198707)43:4<402::aid-jclp2270430411>3.0.co;2-e

Cross, Z. R., Santamaria, A., Corcoran, A. W., Alday, P. M., Coussens, S., and Kohler, M. J. (2018). Alpha oscillations prior to encoding preferentially modulate memory consolidation during wake relative to sleep. bioRxiv [Preprint]. doi: 10.1101/202176

De Bruin, E., Reith, A., Dörflinger, M., and Murer, K. (2011). Feasibility of strengthbalance training extended with computer game dancing in older people; does it affect dual task costs of walking. J. Nov. Physiother. 1:104.

de Bruin, E., and Schmidt, A. (2010). Walking behaviour of healthy elderly: attention should be paid. Behav. Brain Funct. 6:59. doi: 10.1186/1744-9081-659

de Bruin, E., Schoene, D., Pichierri, G., and Smith, S. T. (2010). Use of virtual reality technique for the training of motor control in the elderly Some theoretical considerations. Zeitschrift für Gerontologie und Geriatrie 43, 229-234. doi: 10. 1007/s00391-010-0124-7

de Bruin, E. D., Najafi, B., Murer, K., Uebelhart, D., and Aminian, K. (2007). Quantification of everyday motor function in a geriatric population. J. Rehabil. Res. Dev. 44, 417-428.

Delbaere, K., Close, J. C., Heim, J., Sachdev, P. S., Brodaty, H., Slavin, M. J., et al. (2010). A multifactorial approach to understanding fall risk in older people. J. Am. Geriatr. Soc. 58, 1679-1685. doi: 10.1111/j.1532-5415.2010.03017.x

Delorme, A., and Makeig, S. (2004). EEGLAB: an open source toolbox for analysis of single-trial EEG dynamics including independent component analysis. J. Neurosci. Methods 134, 9-21. doi: 10.1016/j.jneumeth.2003.10.009

Donath, L., Roth, R., Hürlimann, C., Zahner, L., and Faude, O. (2016). Pilates vs. balance training in health community-dwelling seniors: a 3-arm, randomized controlled trial. Int. J. Sports Med. 37, 202-210. doi: 10.1055/s-0035-1559695

Dubost, V., Kressig, R. W., Gonthier, R., Herrmann, F. R., Aminian, K., Najafi, B., et al. (2006). Relationships between dual-task related changes in stride velocity and stride time variability in healthy older adults. Hum. Mov. Sci. 25, 372-382. doi: 10.1016/j.humov.2006.03.004

Eggenberger, P., Theill, N., Holenstein, S., Schumacher, V., and de Bruin, E. D. (2015). Multicomponent physical exercise with simultaneous cognitive training to enhance dual-task walking of older adults: a secondary analysis of a 6-month randomized controlled trial with 1-year follow-up. Clin. Intervent. Aging 10, 1711-1732. doi: 10.2147/cia.s91997

Eggenberger, P., Wolf, M., Schumann, M., and de Bruin, E. D. (2016). Exergame and balance training modulate prefrontal brain activity during walking and enhance executive function in older adults. Front. Aging Neurosci. 8:66. doi: 10.3389/fnagi.2016.00066

El-Khoury, F., Cassou, B., Charles, M.-A., and Dargent-Molina, P. (2013). The effect of fall prevention exercise programmes on fall induced injuries in community dwelling older adults: systematic review and meta-analysis of randomised controlled trials. BMJ 347:f6234. doi: 10.1136/bmj.f6234

Franco, M. R., Tong, A., Howard, K., Sherrington, C., Ferreira, P. H., Pinto, R. Z., et al. (2015). Older people's perspectives on participation in physical activity: a systematic review and thematic synthesis of qualitative literature. Br. J. Sports Med. 49, 1268-1276. doi: 10.1136/bjsports-2014-094015

Gerling, K., and Mandryk, R. (2014). Custom-designed motion-based games for older adults: a review of literature in human-computer interaction. Gerontechnology 12, 68-80. doi: 10.4017/gt.2013.12.2.001.00

Gerling, K. M., Schild, J., and Masuch, M. (2011). "Exergaming for elderly: analyzing player experience and performance," in Mensch \& Computer, ed. M. Eibl, (Munich: Oldenbourg Verlag), 401. doi: 10.1524/9783486712742.401
Grandy, T. H., Werkle-Bergner, M., Chicherio, C., Schmiedek, F., Lovden, M., and Lindenberger, U. (2013). Peak individual alpha frequency qualifies as a stable neurophysiological trait marker in healthy younger and older adults. Psychophysiology 50, 570-582. doi: 10.1111/psyp.12043

Gunning-Dixon, F. M., Brickman, A. M., Cheng, J. C., and Alexopoulos, G. S. (2009). Aging of cerebral white matter: a review of MRI findings. Int. J. Geriatr. Psychiatry 24, 109-117. doi: 10.1002/gps.2087

Guralnik, J. M., Ferrucci, L., Pieper, C. F., Leveille, S. G., Markides, K. S., Ostir, G. V., et al. (2000). Lower extremity function and subsequent disability: consistency across studies, predictive models, and value of gait speed alone compared with the short physical performance battery. J. Gerontol. A Biol. Sci. Med. Sci. 55, M221-M231.

Guralnik, J. M., Simonsick, E. M., Ferrucci, L., Glynn, R. J., Berkman, L. F., Blazer, D. G., et al. (1994). A short physical performance battery assessing lower extremity function: association with self-reported disability and prediction of mortality and nursing home admission. J. Gerontol. 49, M85-M94.

Hackney, M. E., and Wolf, S. L. (2014). Impact of Tai Chi Chu'an practice on balance and mobility in older adults: an integrative review of 20 years of research. J. Geriatr. Phys. Ther. 37, 127-135. doi: 10.1519/JPT. 0b013e3182abe784

Hausdorff, J. M., Yogev, G., Springer, S., Simon, E. S., and Giladi, N. (2005). Walking is more like catching than tapping: gait in the elderly as a complex cognitive task. Exp. Brain Res. 164, 541-548. doi: 10.1007/s00221-005-2280-3

Healy, A. F., Kole, J. A., and Bourne, L. E. Jr. (2014). Training principles to advance expertise. Front. Psychol. 5:131. doi: 10.3389/fpsyg.2014.00131

Hester, R. L., Kinsella, G. J., and Ong, B. (2004). Effect of age on forward and backward span tasks. J. Int. Neuropsychol. Soc. 10, 475-481. doi: 10.1017/ s1355617704104037

Hoffmann, K., Sportwiss, D., Hardy, S., Wiemeyer, J., and Gobel, S. (2015). Personalized adaptive control of training load in cardio-exergames - a feasibility study. Games Health J. 4, 470-479. doi: 10.1089/g4h.2014.0073

Holtzer, R., Verghese, J., Xue, X., and Lipton, R. B. (2006). Cognitive processes related to gait velocity: results from the Einstein aging study. Neuropsychology 20, 215-223. doi: 10.1037/0894-4105.20.2.215

Hu, Y.-N., Chung, Y.-J., Yu, H.-K., Chen, Y.-C., Tsai, C.-T., and Hu, G.-C. (2016). Effect of Tai Chi exercise on fall prevention in older adults: systematic review and meta-analysis of randomized controlled trials. Int. J. Gerontol. 10, 131-136. doi: 10.1016/j.ijge.2016.06.002

Ijsselsteijn, W., De Kort, Y., and Poels, K. (2008). The Game Experience Questionnaire. Eindhoven: Technische Universiteit Eindhoven.

Jasper, H. (1958). Report of the committee on methods of clinical examination in electroencephalography. Electroencephalogr. Clin. Neurophysiol. 10, 370-375. doi: 10.1016/0013-4694(58)90053-1

Jian, Y., Winter, D., Ishac, M., and Gilchrist, L. (1993). Trajectory of the body COG and COP during initiation and termination of gait. Gait Posture 1, 9-22. doi: 10.1016/0966-6362(93)90038-3

Jung, D. (2008). Fear of falling in older adults: comprehensive review. Asian Nurs. Res. 2, 214-222. doi: 10.1016/S1976-1317(09)60003-7

Karlsson, M. K., Magnusson, H., von Schewelov, T., and Rosengren, B. E. (2013). Prevention of falls in the elderly - a review. Osteoporos. Int. 24, 747-762. doi: 10.1007/s00198-012-2256-7

Kattenstroth, J.-C., Kalisch, T., Holt, S., Tegenthoff, M., and Dinse, H. R. (2013). Six months of dance intervention enhances postural, sensorimotor, and cognitive performance in elderly without affecting cardio-respiratory functions. Front. Aging Neurosci. 5:5. doi: 10.3389/fnagi.2013.00005

Kent, P. (2013). The evolution of the wechsler memory scale: a selective review. Appl. Neuropsychol. Adult 20, 277-291. doi: 10.1080/09084282.2012.689267

Klimesch, W. (1999). EEG alpha and theta oscillations reflect cognitive and memory performance: a review and analysis. Brain Res. Rev. 29, 169-195. doi: 10.1016/s0165-0173(98)00056-3

König, N., Singh, N., Von Beckerath, J., Janke, L., and Taylor, W. (2014). Is gait variability reliable? An assessment of spatio-temporal parameters of gait variability during continuous overground walking. Gait Posture 39, 615-617. doi: 10.1016/j.gaitpost.2013.06.014

Lampit, A., Valenzuela, M., and Gates, N. J. (2015). Computerized cognitive training is beneficial for older adults. J. Am. Geriatr. Soc. 63, 2610-2612. doi: 10.1111 /jgs. 13825 
Larsen, L. H., Schou, L., Lund, H. H., and Langberg, H. (2013). The physical effect of exergames in healthy elderly-a systematic review. Games Health J. 2, 205-212. doi: 10.1089/g4h.2013.0036

Lauzé, M., Martel, D., Agnoux, A., Sirois, M.-J., Émond, M., Daoust, R., et al. (2018). Feasibility, acceptability and effects of a home-based exercise program using a gerontechnology on physical capacities after a minor injury in community-living older adults: a pilot study. J. Nutr. Health Aging 22, 16-25. doi: 10.1007/s12603-017-0938-8

Law, L. L., Barnett, F., Yau, M. K., and Gray, M. A. (2014). Effects of combined cognitive and exercise interventions on cognition in older adults with and without cognitive impairment: a systematic review. Ageing Res. Rev. 15, 61-75. doi: 10.1016/j.arr.2014.02.008

Lee, D.-K., Kang, M.-H., Lee, T.-S., and Oh, J.-S. (2015). Relationships among the Y balance test, Berg Balance Scale, and lower limb strength in middle-aged and older females. Braz. J. Phys. Ther. 19, 227-234. doi: 10.1590/bjpt-rbf.20 14.0096

Lewis, C., and Rieman, J. (1993). Task-Centered User Interface Design. A Practical Introduction. Boulder, CO: University of Colorado.

Liukkonen, T. N., Mäkilä, T., Ahtosalo, H., Heinonen, T., Raitoharju, R., and Pitkäkangas, P. (2015). Motion tracking exergames for elderly users. IADIS Int. J. Comput. Sci. Inf. Syst., 52-64.

Maillot, P., Perrot, A., and Hartley, A. (2012). Effects of interactive physical-activity video-game training on physical and cognitive function in older adults. Psychol. Aging 27, 589-600. doi: 10.1037/a0026268

Masud, T., and Morris, R. O. (2001). Epidemiology of falls. Age. Ageing 30(Suppl. 4), 3-7.

McDowd, J. M. (1986). The effects of age and extended practice on divided attention performance. J. Gerontol. 41, 764-769. doi: 10.1093/geronj/41.6.764

Merriman, N. A., Roudaia, E., Romagnoli, M., Orvieto, I., and Newell, F. N. (2018). Acceptability of a custom-designed game, CityQuest, aimed at improving balance confidence and spatial cognition in fall-prone and healthy older adults. Behav. Inf. Technol. 37, 538-557. doi: 10.1080/0144929x.2018.1462402

Mirelman, A., Herman, T., Brozgol, M., Dorfman, M., Sprecher, E., Schweiger, A., et al. (2012). Executive function and falls in older adults: new findings from a five-year prospective study link fall risk to cognition. PLoS One 7:e40297. doi: 10.1371/journal.pone. 0040297

Moher, D., Hopewell, S., Schulz, K. F., Montori, V., Gøtzsche, P. C., Devereaux, P., et al. (2010). CONSORT 2010 explanation and elaboration: updated guidelines for reporting parallel group randomised trials. BMJ 340:c869. doi: 10.1136/bmj. c869

Moore, C. G., Carter, R. E., Nietert, P. J., and Stewart, P. W. (2011). Recommendations for planning pilot studies in clinical and translational research. Clin. Transl. Sci. 4, 332-337. doi: 10.1111/j.1752-8062.2011.00347.x

National Institutes of Health (2011). Global health and aging. National Institutes of Health Publication 11:7737.

Nawaz, A., Skjaeret, N., Helbostad, J. L., Vereijken, B., Boulton, E., and Svanaes, D. (2016). Usability and acceptability of balance exergames in older adults: a scoping review. Health Inform. J. 22, 911-931. doi: 10.1177/1460458215598638

Nelson, M. E., Rejeski, W. J., Blair, S. N., Duncan, P. W., Judge, J. O., King, A. C., et al. (2007). Physical activity and public health in older adults: recommendation from the American College of Sports Medicine and the American Heart Association. Circulation 116, 10942-1105.

Nyman, S. R., and Victor, C. R. (2012). Older people's participation in and engagement with falls prevention interventions in community settings: an augment to the Cochrane systematic review. Age. Ageing 41, 16-23. doi: 10. 1093/ageing/afr 103

Ogawa, E. F., You, T., and Leveille, S. G. (2016). Potential benefits of exergaming for cognition and dual-task function in older adults: a systematic review. J. Aging Phys. Act. 24, 332-336. doi: 10.1123/japa.2014-0267

Okubo, Y., Schoene, D., and Lord, S. R. (2016). Step training improves reaction time, gait and balance and reduces falls in older people: a systematic review and meta-analysis. Br. J. Sports Med. 51, 586-593. doi: 10.1136/bjsports-2015095452

Panel on Prevention of Falls in Older Persons, American Geriatrics Society, and British Geriatrics Society. (2011). Summary of the updated American Geriatrics Society/British Geriatrics Society clinical practice guideline for prevention of falls in older persons. J. Am. Geriatr. Soc. 59, 148-157. doi: 10.1111/j.1532-5415. 2010.03234.x
Paterson, D. H., Jones, G. R., and Rice, C. L. (2007). Ageing and physical activity: evidence to develop exercise recommendations for older adults. Appl. Physiol. Nutr. Metab. 32, S69-S108.

Peel, N. M. (2011). Epidemiology of falls in older age. Can. J. Aging 30, 7-19. doi: $10.1017 / \mathrm{S} 071498081000070 \mathrm{X}$

Peña-Casanova, J., Quiñones-Úbeda, S., Quintana-Aparicio, M., Aguilar, M., Badenes, D., Molinuevo, J. L., et al. (2009). Spanish Multicenter Normative Studies (NEURONORMA Project): norms for verbal span, visuospatial span, letter and number sequencing, trail making test, and symbol digit modalities test. Arch. Clin. Neuropsychol. 24, 321-341. doi: 10.1093/arclin/acp038

Pichierri, G., Murer, K., and de Bruin, E. D. (2012). A cognitive-motor intervention using a dance video game to enhance foot placement accuracy and gait under dual task conditions in older adults: a randomized controlled trial. $B M C$ Geriatrics 12:74. doi: 10.1186/1471-2318-12-74

Pichierri, G., Wolf, P., Murer, K., and de Bruin, E. D. (2011). Cognitive and cognitive-motor interventions affecting physical functioning: a systematic review. BMC Geriatrics 11:29. doi: 10.1186/1471-231811-29 doi: $10.1017 /$ cbo 9780511526824.004

Pirovano, M., Surer, E., Mainetti, R., Lanzi, P. L., and Borghese, N. A. (2016). Exergaming and rehabilitation: a methodology for the design of effective and safe therapeutic exergames. Entertain. Comput. 14, 55-65. doi: 10.1016/j. entcom.2015.10.002

Plisky, P. J., Gorman, P. P., Butler, R. J., Kiesel, K. B., Underwood, F. B., and Elkins, B. (2009). The reliability of an instrumented device for measuring components of the star excursion balance test. N. Am. J. Sports Phys. Ther. 4, 92-99.

Reitan, R. M. (1958). Validity of the trail making test as an indicator of organic brain damage. Percept. Mot. Skills 8, 271-276. doi: 10.2466/pms.1958. 8.3.271

Rikli, R. E., and Jones, C. J. (1999). Development and validation of a functional fitness test for community-residing older adults. J. Aging Phys. Act. 7, 129-161. doi: 10.1093/geront/gns071

Rossini, P. M., Rossi, S., Babiloni, C., and Polich, J. (2007). Clinical neurophysiology of aging brain: from normal aging to neurodegeneration. Prog. Neurobiol. 83, 375-400. doi: 10.1016/j.pneurobio.2007.07.010

Rubenstein, L. Z. (2006). Falls in older people: epidemiology, risk factors and strategies for prevention. Age. Ageing 35(Suppl. 2), ii37-ii41. doi: 10.1093/ ageing/afl084

Schättin, A., Arner, R., Gennaro, F., and de Bruin, E. D. (2016). Adaptations of prefrontal brain activity, executive functions, and gait in healthy elderly following exergame and balance training: a randomizedcontrolled study. Front. Aging Neurosci. 8:278. doi: 10.3389/fnagi.2016. 00278

Schoene, D., Lord, S. R., Delbaere, K., Severino, C., Davies, T. A., and Smith, S. T. (2013). A randomized controlled pilot study of home-based step training in older people using videogame technology. PLoS One 8:e57734. doi: 10.1371/ journal.pone. 0057734

Schoene, D., Valenzuela, T., Lord, S. R., and de Bruin, E. D. (2014). The effect of interactive cognitive-motor training in reducing fall risk in older people: a systematic review. BMC Geriatrics 14:107. doi: 10.1186/1471-2318$14-107$

Schutzer, K. A., and Graves, B. S. (2004). Barriers and motivations to exercise in older adults. Prev. Med. 39, 1056-1061. doi: 10.1016/j.ypmed.2004.04.003

Segev-Jacubovski, O., Herman, T., Yogev-Seligmann, G., Mirelman, A., Giladi, N., and Hausdorff, J. M. (2011). The interplay between gait, falls and cognition: can cognitive therapy reduce fall risk? Expert Rev. Neurother. 11, 1057-1075. doi: 10.1586/ern.11.69

Shaffer, S. W., Teyhen, D. S., Lorenson, C. L., Warren, R. L., Koreerat, C. M., Straseske, C. A., et al. (2013). Y-balance test: a reliability study involving multiple raters. Mil. Med. 178, 1264-1270. doi: 10.7205/MILMED-D-1300222

Sherrington, C., Tiedemann, A., Fairhall, N., Close, J. C., and Lord, S. R. (2011). Exercise to prevent falls in older adults: an updated meta-analysis and best practice recommendations. N. S. W. Public Health Bull. 22, 78-83. doi: 10.1071/ NB10056

Sherrington, C., Whitney, J. C., Lord, S. R., Herbert, R. D., Cumming, R. G., and Close, J. C. T. (2008). Effective exercise for the prevention of falls: a systematic review and meta-analysis. J. Am. Geriatr. Soc. 56, 2234-2243. doi: 10.1111/j. 1532-5415.2008.02014.x 
Shin, S.-S., and An, D.-H. (2015). Comparison of energy expenditure during the Y-balance test in older adults with different visual acuities. J. Phys. Ther. Sci. 27, 697-699. doi: 10.1589/jpts.27.697

Sjösten, N. M., Salonoja, M., Piirtola, M., Vahlberg, T. J., Isoaho, R., Hyttinen, H. K., et al. (2007). A multifactorial fall prevention programme in the communitydwelling aged: predictors of adherence. Eur. J. Public Health 17, 464-470. doi: 10.1093/eurpub/ckl272

Skjæret, N., Nawaz, A., Morat, T., Schoene, D., Helbostad, J. L., and Vereijken, B. (2016). Exercise and rehabilitation delivered through exergames in older adults: an integrative review of technologies, safety and efficacy. Int. J. Med. Inf. 85, 1-16. doi: 10.1016/j.ijmedinf.2015.10.008

Smith, G. L., Banting, L., Eime, R., O’Sullivan, G., and Van Uffelen, J. G. (2017). The association between social support and physical activity in older adults: a systematic review. Int. J. Behav. Nutr. Phys. Act. 14:56.

Solloway, M. R., Taylor, S. L., Shekelle, P. G., Miake-Lye, I. M., Beroes, J. M., Shanman, R. M., et al. (2016). An evidence map of the effect of Tai Chi on health outcomes. Syst. Rev. 5:126. doi: 10.1186/s13643-016-0300-y

Stanmore, E., Stubbs, B., Vancampfort, D., de Bruin, E. D., and Firth, J. (2017). The effect of active video games on cognitive functioning in clinical and nonclinical populations: a meta-analysis of randomized controlled trials. Neurosci. Biobehav. Rev. 78, 34-43. doi: 10.1016/j.neubiorev.2017.04.011

Strauss, E., Sherman, E., and Spreen, O. (1991). A Compendium of Neuropsychological Tests, 3 Edn. New York, NY: Oxford University Press.

Strauss, E., Sherman, E. M., and Spreen, O. (2006). A Compendium of Neuropsychological Tests: Administration, Norms, and Commentary. Washington, DC: American Chemical Society.

Tabei, K.-I., Satoh, M., Ogawa, J.-I., Tokita, T., Nakaguchi, N., Nakao, K., et al. (2017). Physical exercise with music reduces gray and white matter loss in the frontal cortex of elderly people: the Mihama-Kiho scan project. Front. Aging Neurosci. 9:174. doi: 10.3389/fnagi.2017.00174

Taylor, M. E., Ketels, M. M., Delbaere, K., Lord, S. R., Mikolaizak, A. S., and Close, J. C. (2012). Gait impairment and falls in cognitively impaired older adults: an explanatory model of sensorimotor and neuropsychological mediators. Age. Ageing 41, 665-669. doi: 10.1093/ageing/afs057

Thabane, L., Ma, J., Chu, R., Cheng, J., Ismaila, A., Rios, L. P., et al. (2010). A tutorial on pilot studies: the what, why and how. BMC Med. Res. Methodol. 10:1. doi: 10.1186/1471-2288-10-1

Troyer, A. K., Leach, L., and Strauss, E. (2006). Aging and response inhibition: normative data for the Victoria Stroop test. Aging Neuropsychol. Cogn. 13, 20-35. doi: 10.1080/138255890968187

Valenzuela, T., Okubo, Y., Woodbury, A., Lord, S. R., and Delbaere, K. (2018). Adherence to technology-based exercise programs in older adults: a systematic review. J. Geriatric Phys. Ther. 41, 49-61. doi: 10.1519/JPT.0000000000000095

Valiani, V., Lauzé, M., Martel, D., Pahor, M., Manini, T., Anton, S., et al. (2017). A new adaptive home-based exercise technology among older adults living in nursing home: a pilot study on feasibility, acceptability and physical performance. J. Nutr. Health Aging 21, 819-824. doi: 10.1007/s12603-0160820-0

Van Diest, M., Lamoth, C. J., Stegenga, J., Verkerke, G. J., and Postema, K. (2013). Exergaming for balance training of elderly: state of the art and future developments. J. Neuroeng. Rehabil. 10:101. doi: 10.1186/1743-0003-10-101

Van Diest, M., Stegenga, J., Wörtche, H., Verkerke, G. J., Postema, K., and Lamoth, C. (2016). Exergames for unsupervised balance training at home: a pilot study in healthy older adults. Gait Posture 44, 161-167. doi: 10.1016/j.gaitpost.2015. 11.019 van Het Reve, E., Silveira, P., Daniel, F., Casati, F., and de Bruin, E. D. (2014). Tablet-based strength-balance training to motivate and improve adherence to exercise in independently living older people: part 2 of a phase II preclinical exploratory trial. J. Med. Internet Res. 16, e159-e159. doi: 10.2196/jmir.3055

Vaziri, D. D., Aal, K., Ogonowski, C., Von Rekowski, T., Kroll, M., Marston, H. R., et al. (2016). Exploring user experience and technology acceptance for a fall prevention system: results from a randomized clinical trial and a living lab. Eur. Rev. Aging Phys. Act. 13:6. doi: 10.1186/s11556-016-0165-z

Vecchio, F., Babiloni, C., Lizio, R., Fallani, F. D. V., Blinowska, K., Verrienti, G., et al. (2013). "Resting state cortical EEG rhythms in Alzheimer's disease: toward EEG markers for clinical applications: a review," in Supplements to Clinical Neurophysiology, eds E. Başar, C. Başar-Eroglu, A. Özerdem, P. M. Rossini, and G. G. Yener, (Amsterdam: Elsevier), 223-236. doi: 10.1016/b978-0-7020-53078.00015-6

Wang, R.-Y., Wang, Y.-L., Cheng, F.-Y., Chao, Y.-H., Chen, C.-L., and Yang, Y.-R. (2017). Effects of a multicomponent exercise on dual-task performance and executive function among older adults. Int. J. Gerontol. 12, 133-138. doi: 10.1016/j.ijge.2018.01.004

Wechsler, D. (1987). Wechsler Memory Scale-Revised (WMS-R). San Antonio, TX: Psychological Corporation.

Willett, W. C., Koplan, J. P., Nugent, R., Dusenbury, C., Puska, P., and Gaziano, T. A. (2006). "Prevention of chronic disease by means of diet and lifestyle changes," in Disease Control Priorities in Developing Countries, 2nd Edn, eds D. T. Jamison, J. G. Breman, A. R. Measham, G. Alleyne, M. Claeson, D. B. Evans, et al. (Washington, DC: The World Bank).

Wuest, S., Borghese, N. A., Pirovano, M., Mainetti, R., van de Langenberg, R., and de Bruin, E. D. (2014). Usability and effects of an exergame-based balance training program. Games Health J. 3, 106-114. doi: 10.1089/g4h.2013. 0093

Xu, D. Q., Hong, Y., and Li, J. X. (2008). "Tai Chi exercise and muscle strength and endurance in older people," in Medicine and Sport Science, Vol. 52, ed. Y. L. Hong, (Basel: Karger), 20-29. doi: 10.1159/000134281

Yardley, L., Bishop, F. L., Beyer, N., Hauer, K., Kempen, G. I., Piot-Ziegler, C., et al. (2006). Older people's views of falls-prevention interventions in six European countries. Gerontologist 46, 650-660. doi: 10.1093/geront/46. 5.650

Zhao, Y., and Wang, Y. (2016). Tai Chi as an intervention to reduce falls and improve balance function in the elderly: a meta-analysis of randomized controlled trials. Chin. Nurs. Res. 3, 28-33. doi: 10.1016/j.cnre.2015.10.003

Zimmermann, P., and Fimm, B. (2002). "A test battery for attentional performance," in Applied Neuropsychology of Attention. Theory, Diagnosis and Rehabilitation, eds M. Leclercq, and P. Zimmermann, (Hove: Psychology press), $110-151$.

Conflict of Interest: The authors declare that the research was conducted in the absence of any commercial or financial relationships that could be construed as a potential conflict of interest.

Copyright (c) 2019 Adcock, Thalmann, Schättin, Gennaro and de Bruin. This is an open-access article distributed under the terms of the Creative Commons Attribution License (CC BY). The use, distribution or reproduction in other forums is permitted, provided the original author(s) and the copyright owner(s) are credited and that the original publication in this journal is cited, in accordance with accepted academic practice. No use, distribution or reproduction is permitted which does not comply with these terms. 